\title{
Stellar Black Hole Binary Mergers in Open Clusters
}

\author{
S. Rastello ${ }^{1 \star}$, P. Amaro-Seoane ${ }^{2}$, M. Arca-Sedda ${ }^{1,3}$, R. Capuzzo-Dolcetta ${ }^{1}$, \\ G. Fragione ${ }^{4}$, I. Tosta e Melo 1 \\ ${ }^{1}$ Dep. of Physics, Sapienza, Universitá di Roma, P.le A. Moro 5, 00185, Roma, Italy \\ ${ }^{2}$ Institute of Space Sciences (ICE, CSIC) \& Institut d'Estudis Espacials de Catalunya (IEEC) \\ at Campus UAB, Carrer de Can Magrans s/n 08193 Barcelona, Spain \\ Institute of Applied Mathematics, Academy of Mathematics and Systems Science, CAS, Beijing 100190, China \\ Kavli Institute for Astronomy and Astrophysics, Beijing 100871, China \\ Zentrum für Astronomie und Astrophysik, TU Berlin, Hardenbergstraße 36, 10623 Berlin, Germany \\ ${ }^{3}$ Astronomisches Rechen-Institut, Mönchhofstraße 12-14, 69120 Heidelberg, Germany \\ ${ }^{4}$ Hebrew University of Jerusalem, Racah Institute of Physics, Givat Ram, 9190401, Jerusalem, Israel
}

Accepted XXX. Received YYY; in original form ZZZ

\begin{abstract}
In this paper we study the evolution of a primordial black hole binary (BHB) in a sample of over 1500 direct-summation $N$-body simulations of small-and intermediatesize isolated star clusters as proxies of galactic open clusters. The BHBs have masses in the range of the first LIGO/Virgo detections. Some of our models show a significant hardening of the BHB in a relatively short time. Some of them merge within the cluster, while ejected binaries, typically, have exceedingly long merger timescales. The perturbation of stars around BHB systems is key to induce their coalescence. The BHBs which merge in the cluster could be detected with a delay of a few years between space detectors, as future LISA, and ground-based ones, due to their relatively high eccentricity. Under our assumptions, we estimate a BHB merger rate of $R_{\mathrm{mrg}} \sim 2 \mathrm{yr}^{-1}$ $\mathrm{Gpc}^{-3}$. We see that in many cases the BHB triggers tidal disruption events which, however, are not linked to the GW emission. Open cluster-like systems are, hence, a promising environment for GWs from BHBs and tidal disruptions.
\end{abstract}

Key words: Galaxy: open clusters and associations: general - stars: black holes stars: kinematics and dynamics - gravitational waves

\section{INTRODUCTION}

The Laser Interferometer Gravitational-Wave Observatory (LIGO) and Virgo have detected six sources of GWs as of writing this article (Abbott et al. 2016a,b, 2017a; The LIGO Scientific Collaboration et al. 2017; Abbott et al. 2017b,c). All of them but one, GW170817, last reference, correspond to a system of BHB. Typically, the masses are larger than the nominal one derived from stellar evolution of $10 M_{\odot}$, as predicted by Amaro-Seoane \& Chen (2016), i.e. "hyperstellar-mass black holes", as the authors coined, and previously discussed by e.g. also Heger et al. 2003; Mapelli et al. 2008; Zampieri \& Roberts 2009; Mapelli et al. 2010; Belczynski et al. 2010a; Fryer et al. 2012; Mapelli \& Bressan 2013; Ziosi et al. 2014; Spera et al. 2015.

There are two different channels to form a BHB, namely either (i) in the field in isolation and via stellar evolution of

^ E-mail: sara.rastello@uniroma1.it a binary of two extended stars (see e.g. Tutukov \& Yungelson 1973; Bethe \& Brown 1998; Belczynski et al. 2002, 2010b; Postnov \& Yungelson 2014; Loeb 2016; Tutukov \& Cherepashchuk 2017; Postnov \& Kuranov 2017; Giacobbo \& Mapelli 2018).

(ii) or via dynamical interactions in a dense stellar system (see the review of Benacquista \& Downing 2013 and e.g. also Banerjee et al. 2010; Downing et al. 2010; Mapelli \& Bressan 2013; Ziosi et al. 2014; Rodriguez et al. 2015, 2016a; Mapelli 2016; Askar et al. 2017; Antonini et al. 2016; Arca-Sedda \& Capuzzo-Dolcetta 2017a; Fragione \& Kocsis 2018).

In this article we address the evolution of a BHB in an open cluster with a suit of 1500 direct-summation $N$-body simulations. We model the evolution of the BHB with properties similar to what can be expected to be detected by LIGO/Virgo (Amaro-Seoane \& Chen 2016), using different representations of small- and intermediate-mass isolated open star clusters.

Our cluster models are considered as a proxy of the 
Galactic population of open clusters, which are characterized by central densities of a few $\mathrm{M}_{\odot} \mathrm{pc}^{-3}$, i.e. much lower than the typical densities of globular clusters, and contain a number of stars from two to three orders of magnitude less than a globular cluster.

We investigate the evolution of stellar BHBs in low-mass and low-density open clusters models by means of high precision direct-summation $N$-body simulations. In an open cluster the impulsive effect produced by the large fluctuations over the mean field, whose amplitude is of order $\sqrt{N} / N$, can significantly affect the BHB evolution. Assuming an initial number of star $N_{\mathrm{O}}=1000$ for this type of open clusters and $N_{\mathrm{g}}=10^{6}$ for a typical globular cluster, we can calculate the expected fluctuations over the mean field amplitude as $f=\sqrt{N_{\mathrm{g}} / N_{\mathrm{o}}}=\sqrt{1000} \simeq 32$, thus implying a larger such effect in open clusters. This enhanced effect of stochastic fluctuations (physically given by the rare but close approaches among cluster stars) reflects in the ratio of the 2-body relaxation time scales which, given the cluster sizes as $R_{\mathrm{O}}$ and $R_{\mathrm{g}}$, writes as (Spitzer 1987)

$\frac{t_{\mathrm{rlx}, \mathrm{o}}}{t_{\mathrm{rlx}, \mathrm{g}}}=\frac{1}{f} \frac{\log \left(0.11 N_{\mathrm{o}}\right)}{\log \left(0.11 N_{\mathrm{g}}\right)}\left(\frac{R_{\mathrm{O}}}{R_{\mathrm{g}}}\right)^{3 / 2}$.

Assuming $R_{\mathrm{O}} / R_{\mathrm{g}}=1 / 5$, the above equation yields to $t_{\mathrm{rlx}, \mathrm{o}} / t_{\mathrm{rlx}, \mathrm{g}} \simeq 0.02$, meaning that the smaller system evolves 50 times faster. Of course, this enhanced effect of individual strong encounters is partly compensated by their smaller time rate.

In this paper we address the evolution of a BHB which, as a result of dynamical friction orbital decay (see e.g. Binney \& Tremaine 2008), we assume to be located at the centre of an open cluster-like system. The masses of the black holes are set to $30 M_{\odot}$ each, following the first LIGO/Virgo detection, the GW150914 source (Abbott et al. 2016a), and Amaro-Seoane \& Chen (2016). Despite the possible ejection due to the supernova natal kick, there is margin for such kind of remnant to be retained in an open cluster. Indeed, compact remnants such as neutron stars and black holes formed in massive binaries are much easier retained in clusters because the kick momentum is shared with a massive companion, which leads to a much lower velocity for the post-supernova binary (Podsiadlowski et al. 2004, 2005). In the case of neutron stars, Podsiadlowski et al. (2004) showed that for periods below 100 days, the supernova explosion leads to a very little or no natal kick at all (their Fig.2, the dichotomous kick scenario). Open clusters have binaries mostly with periods of 100 days and below (see Mathieu 2008, based on the data of Duquennoy \& Mayor 1991). These results can be extrapolated to black holes because they receive a similar kick to neutron stars (see Repetto et al. 2012 and also the explanation of Janka 2013 of this phenomenon). In any case, black holes with masses greater than $10 M_{\odot}$ at solar metallicity form via direct collapse and do not undergo supernova explosion, and hence do not receive a natal kick (Perna et al. 2018). Also, while the solar metallicity in principle could not lead to the formation of black holes more massive than $25 M_{\odot}$ Spera \& Mapelli (2017), we note that the resonant interaction of two binary systems can lead to a collisional merger which leads to formation of this kind of black hole (Goswami et al. 2014; Fregeau et al. 2004) at the centre of a stellar system, where they naturally segregate due to dynamical friction.

Moreover, we note that another possibility is that these stellar-mass black holes could have got their large masses due to repeated relativistic mergers of lighter progenitors. However, the relativistic recoil velocity is around $200-450 \mathrm{~km} / \mathrm{s}$ for progenitors with mass ratio $\sim[0.2,1]$ respectively, so that this possibility is unlikely (see e.g. Amaro-Seoane \& Chen 2016, their Fig. 1, lower panel), because they would escape the cluster, unless the initial distribution of spins is peaked around zero and the black holes have the same mass (as in the work of Rodriguez et al. (2018) in the context of globular clusters). In this case, second generation mergers are possible and, hence, one can form a more massive black hole via successive mergers of lighter progenitors.

The relatively low number of stars of open clusters gives the possibility to integrate over at least a few relaxation times in a relatively short computational time, so that, contrarily to the cases of globular clusters or galactic nuclei, it is possible to fully integrate these systems without the need to rescale the results.

In this article we present a series of 1500 dedicated direct-summation $N$-body simulations of open clusters with BHBs. The paper is organized as follows: in Sect. 2 we describe the numerical methods used and our set of models; in Sect. 3 we present and discuss the results of the BHB dynamics; in Sect. 4 we discuss the implication of our BHBs as sources of gravitational waves; in Sect. 5 we present the results on tidal disruption events, in Sect. 6 we draw overall conclusions.

\section{METHOD AND MODELS}

To study the BHB evolution inside its parent open cluster (henceforth OC) we used NBODY7 (Aarseth 2012), a direct $\mathrm{N}$-body code that integrates in a reliable way the motion of stars in stellar systems, and implements a careful treatment to deal with strong gravitational encounters, taking also into account stellar evolution. We performed several simulations at varying both $\mathrm{OC}$ and $\mathrm{BHB}$ main properties, taking advantage of the two high-performance workstations hosted at Sapienza, University of Roma, and the Kepler cluster, hosted at the Heidelberg University.

Table 1 summarizes the main properties of our $N$-Body simulation models. We created four simulation groups representing OC models at varying initial number of particles, namely $512 \leq N \leq 4096$. Assuming a Kroupa (2001) initial mass function $\left(0.01 \mathrm{M}_{\odot} \leq \mathrm{M} \leq 100 \mathrm{M}_{\odot}\right)$, our OC model masses range between $300 \mathrm{M}_{\odot}$ and $3000 \mathrm{M}_{\odot}$. All clusters are modeled according to a Plummer density profile (Plummer 1911) at virial equilibrium with a core radius $\left(r_{c}=1\right.$ $\mathrm{pc}$ ), and adopting solar metallicity $\left(\mathrm{Z}_{\odot}\right)$. We perform all the simulations including the stellar evolution recipes that are implemented in the NBODY7 code which come from the standard SSE and BSE tools (Hurley et al. 2000, 2002), with updated stellar mass loss and remnant formation prescriptions from Belczynski et al. (2010c). Further, for simplicity, we do not take into account primordial binaries, which we leave to future work. To give statistical significance to the results we made 150 different realizations of every model, which are denoted with names A00, A05, B00, B05, C00, 
Table 1. Main parameters characterizing our models. The first two columns refers to the cluster total number of stars, $N_{\mathrm{cl}}$, and its mass $\boldsymbol{M}_{\mathrm{cl}}$. The second two-column group refers to the BHB parameters: semi-major axis, $a$, and initial eccentricity, $e$. The last column gives the model identification name. Each model is comprised of 150 different OC realizations.

\begin{tabular}{ccccc}
\hline \multicolumn{2}{c}{ Cluster } & \multicolumn{2}{c}{ BHB } & $N$-body set \\
\hline$N_{\mathrm{cl}}$ & $M_{\mathrm{cl}}\left(\mathrm{M}_{\odot}\right)$ & $a(\mathrm{pc})$ & $e$ & Model \\
\hline 512 & \multirow{2}{*}{$3.2 \times 10^{2}$} & \multirow{2}{*}{0.01} & 0.0 & $\mathrm{~A} 00$ \\
& & 0.5 & $\mathrm{~A} 05$ \\
\hline \multirow{2}{*}{7024} & \multirow{2}{*}{$7.1 \times 10^{2}$} & \multirow{2}{*}{0.01} & 0.0 & $\mathrm{~B} 00$ \\
& & \multirow{2}{*}{0.01} & 0.0 & $\mathrm{~B} 05$ \\
\hline \multirow{2}{*}{$1.4 \times 10^{3}$} & \multirow{2}{*}{0.01} & 0.5 & $\mathrm{C} 00$ \\
& \multirow{2}{*}{$2.7 \times 10^{3}$} & \multirow{2}{*}{0.01} & 0.0 & $\mathrm{D} 05$ \\
\hline
\end{tabular}

C05, D00 and D05, where the letter refers to increasing $N$ and the digits to the initial BHB orbital eccentricity. Additionally, we ran a further sample of 421 simulations, aiming at investigating the implications of some of our assumptions on the BHB evolution. These models are deeply discussed in Sect. 5 .

In all our simulations, we assumed that the BHB is initially placed at the centre of its host $\mathrm{OC}$, and is composed of two equal mass BHs with individual mass $\mathrm{M}_{\mathrm{BH}}=30 \mathrm{M}_{\odot}$. The initial BHB semi major axis is $0.01 \mathrm{pc}$ with two initial eccentricities, $e_{\mathrm{BHB}}=0, e_{\mathrm{BHB}}=0.5$. The initial conditions drawn this way are obtained updating the procedure followed in Arca-Sedda et al. (2015). The choice of a BHB initially at rest at centre of the cluster with that not very small separation is not a limitation because the dynamical friction time scale of $30 \mathrm{M}_{\odot}$ is short enough to make likely that both the orbital decay of the BHB occurs rapidly and also that the probability of a rapid formation of a BHB from two individual massive $\mathrm{BHs}$ is large even on a short time.

The BHB orbital period is, for the given choices of masses and semimajor axis, $\mathrm{P}_{\mathrm{BHB}}=0.012 \mathrm{Myr}$. Note that our BHBs are actually "hard" binaries (Heggie 1975; Hills 1975; Binney \& Tremaine 2008), having binding energy BE $3.810^{45} \mathrm{erg}$, which is larger than the average kinetic energy of the field stars in each type of cluster studied in this work. All models were evolved up to $3 \mathrm{Gyr}$, which is about 3 times the simulated OC internal relaxation time. The scope of the present work is to give investigate the BHB dynamical evolution, hence we focus on tracking mainly its evolution. We also note that stellar-mass BHs naturally form binary systems in open clusters over a wide cluster mass range, and can also undergo triple-/subsystem-driven mergers, as recently shown through explicit direct N-body simulations by Kimpson et al. (2016) and Banerjee (2017).

\section{DYNAMICS OF THE BLACK HOLE BINARY}

\subsection{General evolution}

The BHB is assumed to be located at the centre of the cluster. Due to interactions with other stars, the BHB can either undergo one of the following three outcomes. First, (i) the BHB can shrink and hence become harder, meaning that the kinetic energy of the BHB is higher than the average in the system (see e.g. Binney \& Tremaine 2008); also (ii) the BHB
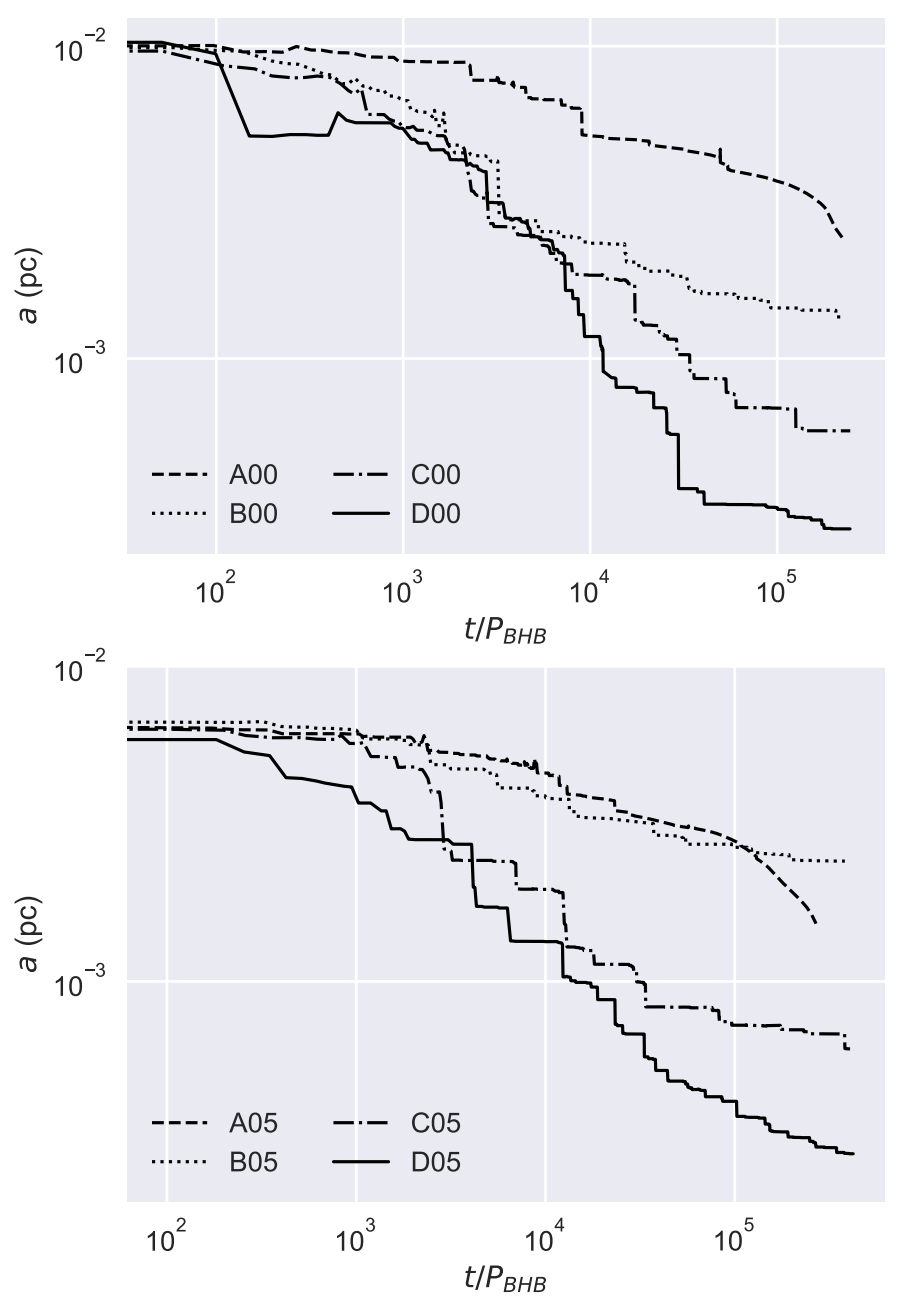

Figure 1. Semi-major axis evolution in four random, representative simulations. The upper panel shows binaries which initially are circular and lower panel depicts eccentric ones. We normalise the time to the initial (i.e. at $T=0$ ) period of the binary.

can gain energy and therefore increase its semi-major axis and (iii) the BHB can be ionised in a typically three-body encounter. In Table 2 we show the percentages of these three outcomes in our simulations. 
Table 2. Percentage of BHB which undergo one of the three processes described in the text. They either shrink (column 2), or increase their semi-major axis (column 3) or break up (column $4)$.

\begin{tabular}{cccc}
\hline Model & $\begin{array}{c}\text { Harder } \\
\%\end{array}$ & $\begin{array}{c}\text { Wider } \\
\%\end{array}$ & $\begin{array}{c}\text { Break up } \\
\%\end{array}$ \\
\hline A00 & 89.1 & 7.9 & 2.9 \\
A05 & 97.1 & 2.1 & 0.7 \\
B00 & 92.5 & 2.7 & 4.8 \\
B05 & 94.0 & 2.0 & 4.0 \\
C00 & 93.6 & 0 & 6.4 \\
C05 & 96.5 & 0 & 3.5 \\
D00 & 94.2 & 0 & 5.8 \\
D05 & 97.1 & 0 & 2.8 \\
\hline
\end{tabular}

We can see that typically about $90 \%$ of all binaries shrink their semi-major axis as they evolve, as one can expect from the so-called Heggie's law (Heggie 1975). We note that models in which the binary initially was eccentric lead to a higher percentage in the "harder" outcome. We display in Fig. 1 a few representative examples of these processes. The decrease is gradual for model A and B while model C and D (which are the more massive) show a steeper decrease.

There are however cases in which the binary gains energy from gravitational encounters and increases its semimajor axis, becoming "wider" (Table 2, column 2). If the host cluster is massive enough, the semi-major axis always decreases (models $\mathrm{C}$ and $\mathrm{D}$ ), contrary to lighter models, in which it can increase (models A and B).

Because of the initial choice of the BHB semi-major axis, gravitational encounters with other stars rarely ionise it, although we observe a few events, typically below $7 \%$ (circular binaries are easier to ionise). This ionisation happens between $\sim 5 \mathrm{Myr}$ and up to $\sim 100 \mathrm{Myr}$ and it is usually driven by the encounter with a massive $\operatorname{star}\left(\gtrsim 10 \mathrm{M}_{\odot}\right)$. In such case, the massive star generally pair with one of the $\mathrm{BHs}$, while the other $\mathrm{BH}$ is usually ejected from the stellar systems.

\subsubsection{Pericentre evolution}

For a BHB to become an efficient source of GWs, the pericentre distance must be short enough. In this section we analyse the evolution of the pericentres for our different models. In Table 3 we summarise the results of Figs. 2, 3. In the table we show the average pericentre distance at three different times (1, 2 and $3 \mathrm{Gyr})$ in the evolution of the cluster as well as the absolute minimum pericentre distance we find at each of these times.

For BHB which initially are circular, we can see in the table and in Fig. 2 that in all models there is a significative shrinkage of the pericentre distance of, at least, one order of magnitude. Such shrinkage occurs after only 1 Gyr. For the most massive clusters, i.e. model D00, about $20 \%$ of all binaries achieve a pericentre distance which is of about two orders of magnitude smaller than the initial value. We note, however, that a very few binaries shrink to extremely small values, reaching pericentre distances of down to $10^{-7} \mathrm{pc}$. Eccentric binaries also shrink and achieve smaller pericentre values, as we can see in Fig. 3. In both the case of eccentric and circular orbit, we note that in low dense clusters, i.e.
Table 3. Evolution of the BHB pericentre distance for all the models. The columns from left to right denote, respectively: the model, the initial BHB pericentre $\left(\mathrm{r}_{\mathrm{p}}^{i}\right)$, the time in which we have calculated the average $(T)$, the BHB pericentre distance averaged over all the simulations of the respective model $\left(\left\langle r_{\mathrm{p}}\right\rangle\right)$, and the absolute minimum distance we record $\left(\mathrm{r}_{\mathrm{p}}^{\text {min }}\right)$. We note that the Schwarzschild radius of a $30 M_{\odot}$ is $1.43 \times 10^{-12} \mathrm{pc}$.

\begin{tabular}{|c|c|c|c|c|}
\hline Model & $\begin{array}{c}\mathbf{r}_{\mathrm{p}}^{i} \\
(\mathrm{pc})\end{array}$ & $\begin{array}{c}\mathbf{T} \\
(\mathrm{Gyr})\end{array}$ & $\begin{array}{l}\left\langle r_{\mathrm{p}}\right\rangle \\
(\mathrm{pc})\end{array}$ & $\begin{array}{c}\mathbf{r}_{\mathrm{p}}^{\min } \\
(\mathrm{pc})\end{array}$ \\
\hline A00 & $1.0 \times 10^{-2}$ & $\begin{array}{l}1 \\
2 \\
3\end{array}$ & $\begin{array}{l}2.3 \times 10^{-3} \\
2.3 \times 10^{-3} \\
2.1 \times 10^{-3}\end{array}$ & $\begin{array}{l}5.0 \times 10^{-6} \\
3.2 \times 10^{-5} \\
4.9 \times 10^{-6}\end{array}$ \\
\hline A 05 & $5.0 \times 10^{-3}$ & $\begin{array}{l}1 \\
2 \\
3\end{array}$ & $\begin{array}{l}1.7 \times 10^{-3} \\
1.9 \times 10^{-3} \\
1.7 \times 10^{-3}\end{array}$ & $\begin{array}{l}1.4 \times 10^{-5} \\
1.0 \times 10^{-5} \\
2.7 \times 10^{-4}\end{array}$ \\
\hline B00 & $1.0 \times 10^{-2}$ & $\begin{array}{l}1 \\
2 \\
3\end{array}$ & $\begin{array}{l}5.4 \times 10^{-4} \\
5.7 \times 10^{-4} \\
5.1 \times 10^{-4}\end{array}$ & $\begin{array}{l}3.4 \times 10^{-6} \\
2.2 \times 10^{-6} \\
4.1 \times 10^{-6}\end{array}$ \\
\hline B05 & $5.0 \times 10^{-3}$ & $\begin{array}{l}1 \\
2 \\
3\end{array}$ & $\begin{array}{l}1.1 \times 10^{-3} \\
8.9 \times 10^{-4} \\
7.9 \times 10^{-4}\end{array}$ & $\begin{array}{l}2.4 \times 10^{-7} \\
2.4 \times 10^{-7} \\
1.5 \times 10^{-6}\end{array}$ \\
\hline $\mathrm{C} 00$ & $1.0 \times 10^{-2}$ & $\begin{array}{l}1 \\
2 \\
3\end{array}$ & $\begin{array}{l}2.8 \times 10^{-4} \\
2.6 \times 10^{-4} \\
2.5 \times 10^{-4}\end{array}$ & $\begin{array}{l}1.7 \times 10^{-6} \\
2.2 \times 10^{-7} \\
5.3 \times 10^{-7}\end{array}$ \\
\hline $\mathrm{C} 05$ & $5.0 \times 10^{-3}$ & $\begin{array}{l}1 \\
2 \\
3\end{array}$ & $\begin{array}{l}3.7 \times 10^{-4} \\
3.1 \times 10^{-4} \\
2.6 \times 10^{-4}\end{array}$ & $\begin{array}{l}1.5 \times 10^{-6} \\
2.5 \times 10^{-7} \\
2.5 \times 10^{-7}\end{array}$ \\
\hline D00 & $1.0 \times 10^{-2}$ & $\begin{array}{l}1 \\
2 \\
3\end{array}$ & $\begin{array}{l}1.3 \times 10^{-4} \\
1.0 \times 10^{-4} \\
9.1 \times 10^{-5}\end{array}$ & $\begin{array}{l}2.7 \times 10^{-6} \\
9.2 \times 10^{-7} \\
8.8 \times 10^{-7}\end{array}$ \\
\hline D05 & $5.0 \times 10^{-3}$ & $\begin{array}{l}1 \\
2 \\
3\end{array}$ & $\begin{array}{l}1.5 \times 10^{-4} \\
1.5 \times 10^{-4} \\
1.3 \times 10^{-4}\end{array}$ & $\begin{array}{l}1.8 \times 10^{-6} \\
3.6 \times 10^{-6} \\
9.8 \times 10^{-6}\end{array}$ \\
\hline
\end{tabular}

model $\mathrm{A}$ and $\mathrm{B}$, the $\mathrm{BHB}$ preserves a pericentre relatively close to the initial value indicating that such stellar systems are less efficient in favouring the BHB shrinkage. In such models the pericentres data appears more spread than in models $\mathrm{C}$ and $\mathrm{D}$. A further difference is that for example in model A00, even after $3 \mathrm{Gyr}$, the BHB have larger pericentres, indicating that the binary becomes wider, contrary to what is observed in model A05. We note additionally, that in the intermediate low massive model, B05, the pericentre reaches very small values (of the order of $10^{-7} p c$ ) which does not occur for an initial circular orbit. These results indicate that in such cases, both the cluster stellar density and the initial orbital eccentricity play a relevant role in favouring the BHB shrinkage.

\subsection{Retained and dynamically-ejected BHBs}

We observe that in the majority of cases these dynamicallyformed binaries interacting with other stars in the system can also be ejected away from the cluster. In the code that we are using, NBODY7, single or binary stars are considered escapers of their energy is positive and their distance to the centre of the OC centre is at least two times the initial half mass radius (Aarseth 1973, 2003). Taking into account 

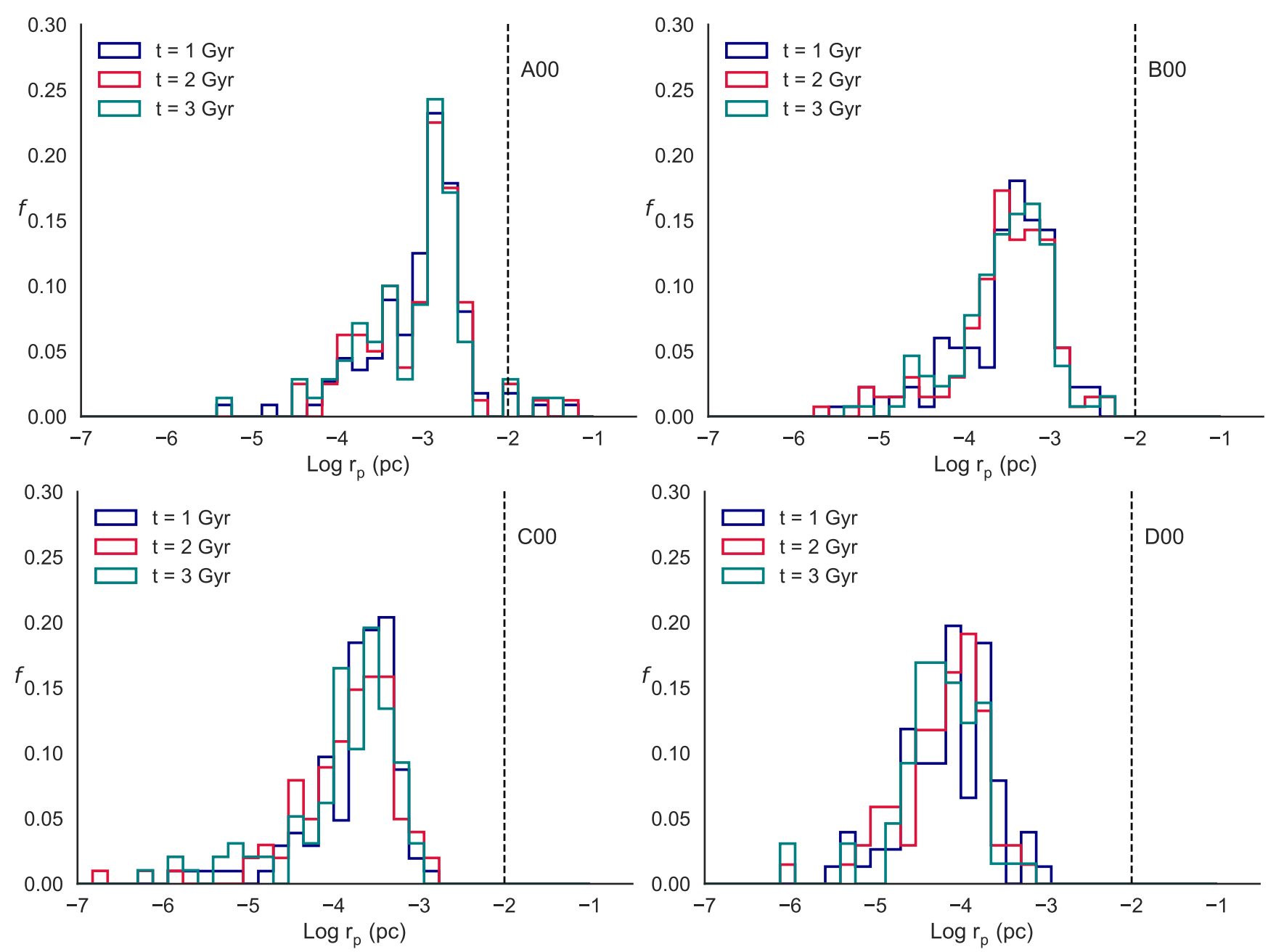

Figure 2. BHB pericentre distance distribution for all simulations of models A00, B00, C00 and D00. The histograms are calculated at three different points in the evolution of the systems, namely at 1 Gyr (blue), 2 Gyr (red) and 3 Gyr (green). We show with a vertical, black dashed line the initial pericentre in the model.

the evolutionary scenarios discussed in Sect. 3.1, we derive for each model the fraction of escaping and retained BHBs. Table 4 summarizes the results of this analysis.

In model A all the BHBs that become harder, i.e. shrink their semi-major axis, are retained in the $\mathrm{OC}$ both in the cases in which the binary has an initial circular orbit (A00) and in the cases in which the BHBs has initial eccentric orbit (A05).

In model B only a small fraction of BHBs $(0.7 \%)$ is ejected from the cluster while a large fraction is retained. In particular, in model B05 the fraction of ejected BHBs (2.7 $\%$ ) is higher than in model B00. In model $\mathrm{C}$ the percentage of ejected BHBs is larger than in the previous cases. In particular, when the binary has an initial eccentric orbit (model C05) the fraction of escaping BHBs is about the $10.5 \%$. Finally, in model D the majority ( $\geq 85 \%$ ) of BHBs is retained in the cluster even if in this case, contrary to the previous situations, circular orbits have a higher fraction of ejected BHBs.

After an ionisation of the BHB, the individual black holes usually form a new binary with a star in the cluster. These dynamically-formed binaries are usually short-lived, and last at most some tens of Myrs.
After a BHB has been separated, one of the black holes stays in the cluster and forms a new binary with a star. These dynamically-formed binaries do not survive for long. Moreover, we notice that the newly single BHs are more likely to be expelled from the stellar systems than retained because of multiple scattering with massive stars $\left(\gtrsim 10 \mathrm{M}_{\odot}\right)$. The presence of such massive stars is comparable to the time at which the BHs are expelled from the systems, which is generally short $(\lesssim 100 \mathrm{Myr})$. As it is shown in Table 4 , only in three models studied (A00, C00 and D00) the BHs are retained after the binary breaking.

Note that the larger fraction of ejected BHBs "belongs" to more massive clusters (C and $\mathrm{D})$ in spite of their larger escape velocity.

The time at which the bound BHB is ejected from the cluster varies among the models, with a BHB mean ejection time between 0.4 and $1.2 \mathrm{Gyr}$. We noticed that low dense clusters (models A and B) show a BHB ejection time shorter than massive clusters (models $\mathrm{C}$ and $\mathrm{D}$ ).

The pie charts in Fig. 4 illustrate the probabilities of the different channels for two models studied, C and D (both configuration 00 and 05 ). Harder binaries are denoted with letter " $h$ ", wider with "w", broken up binaries with " $b$ ". Then, 

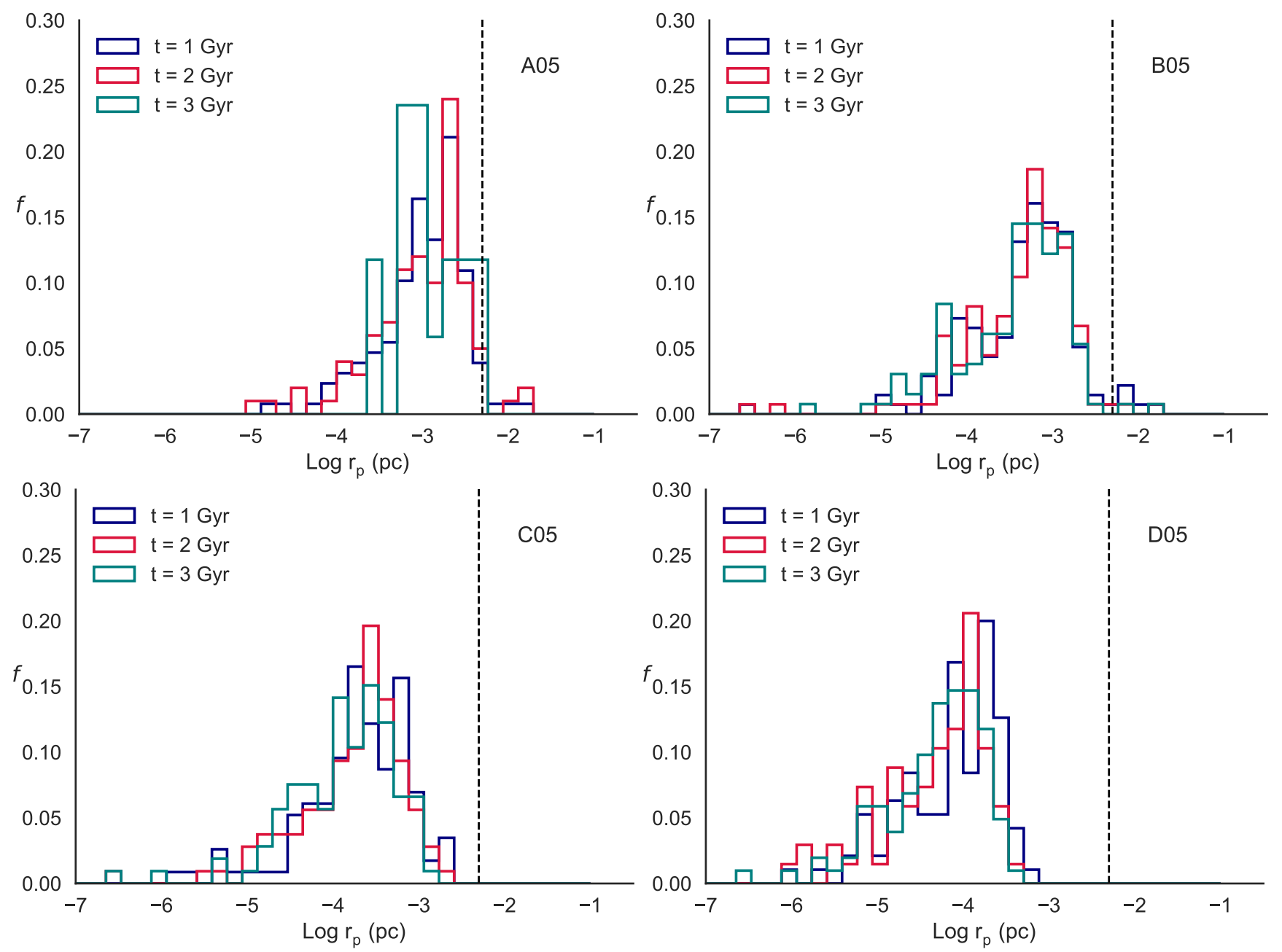

Figure 3. As in Fig.2, but for the models A05, B05, C05 and D05.

Table 4. Percentage of BHBs retained (ret) by the cluster or ejected (esc). The first column indicates the models. Column 2 and 3 indicate the percentage of retained or ejected BHB that become harder. Column 4 and 5 refers to wider BHBs. Column 6 and 7 give the percentage of retained and ejected single black hole after the binary breaking.

\begin{tabular}{ccccccc}
\hline Model & \multicolumn{3}{c}{ hard } & \multicolumn{2}{c}{ wider } & \multicolumn{2}{c}{ break } \\
\hline & ret & esc & ret & esc & ret & esc \\
& $\%$ & $\%$ & $\%$ & $\%$ & $\%$ & $\%$ \\
A00 & 89.1 & 0.0 & 7.9 & 0.0 & 0.7 & 2.1 \\
A05 & 97.1 & 0.0 & 2.1 & 0.0 & 0.0 & 0.7 \\
B00 & 91.8 & 0.7 & 2.7 & 0.0 & 0.0 & 4.8 \\
B05 & 91.3 & 2.7 & 2.0 & 0.0 & 0.0 & 4.0 \\
C00 & 88.6 & 4.9 & 0.0 & 0.0 & 0.7 & 5.6 \\
C05 & 86.5 & 9.9 & 0.0 & 0.0 & 0.0 & 3.5 \\
D00 & 85.5 & 8.6 & 0.0 & 0.0 & 0.7 & 5.0 \\
D05 & 90.0 & 7.2 & 0.0 & 0.0 & 0.0 & 2.8 \\
\hline
\end{tabular}

each of the three scenarios are split into two cases: BHB retained by the cluster (indicate with "ret") and BHB or BHs ejected from the system (indicate with "esc"). From the pie charts it is clear that in the majority of cases the BHBs shrink the semi major axis, becoming harder and remaining bound to the parent cluster. Model C00 and D00 show also a very small fraction of broken up binaries (hence newly single BHs) which are retained by the clusters. Such result, on the contrary, is not observed in model C05 and D05. A considerable number of harder BHB escaped from the cluster is observed in model C05. Furthermore, the percentage of newly single BHs escaped from the cluster $\left(b_{\mathrm{esc}}\right)$ is higher in model C00 and D00. Finally it is worth noticing the fraction of coalescence events (black slices) in each model.

\subsection{External Tidal Field}

For a Milky Way-like galaxy the dynamical evolution of open clusters may be significantly influenced by an external tidal field (Banerjee 2017). To investigate such effect, we assume our clusters are embedded in a tidal field like that of the solar neighbourhood. The Galactic potential is modelled using a bulge mass of $\mathrm{MB}=1.5 \cdot 10^{10} \mathrm{M}_{\odot}($ Miyamoto \& Nagai 1975$)$ and disc mass $\mathrm{MD}=5 \cdot 10^{10} \mathrm{M}_{\odot}$. The geometry of the disc is modelled following the formulae of Dehnen (1993) with the following scale parameters $a=5.0 \mathrm{kpc}$ and $b=0.25 \mathrm{kpc}$. A logarithmic halo is included such that the circular velocity is $220 \mathrm{~km} / \mathrm{s}$ at $8.5 \mathrm{kpc}$ from the Galactic center. Adopting these configurations, we ran a further sub-set of simulations for each model A, B, C and D. The external tidal field generally 


h_ret $\quad$ h_esc w_ret $\quad$ w_esc b_ret $\quad$ b_esc merge
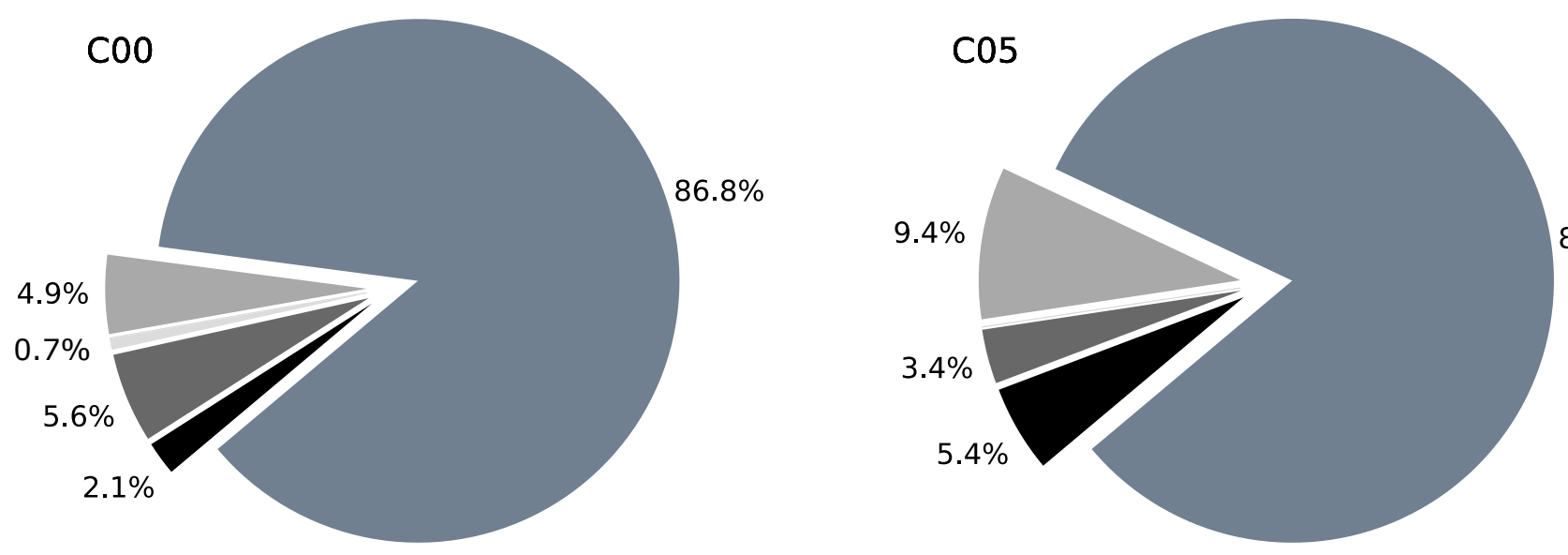

$81.9 \%$
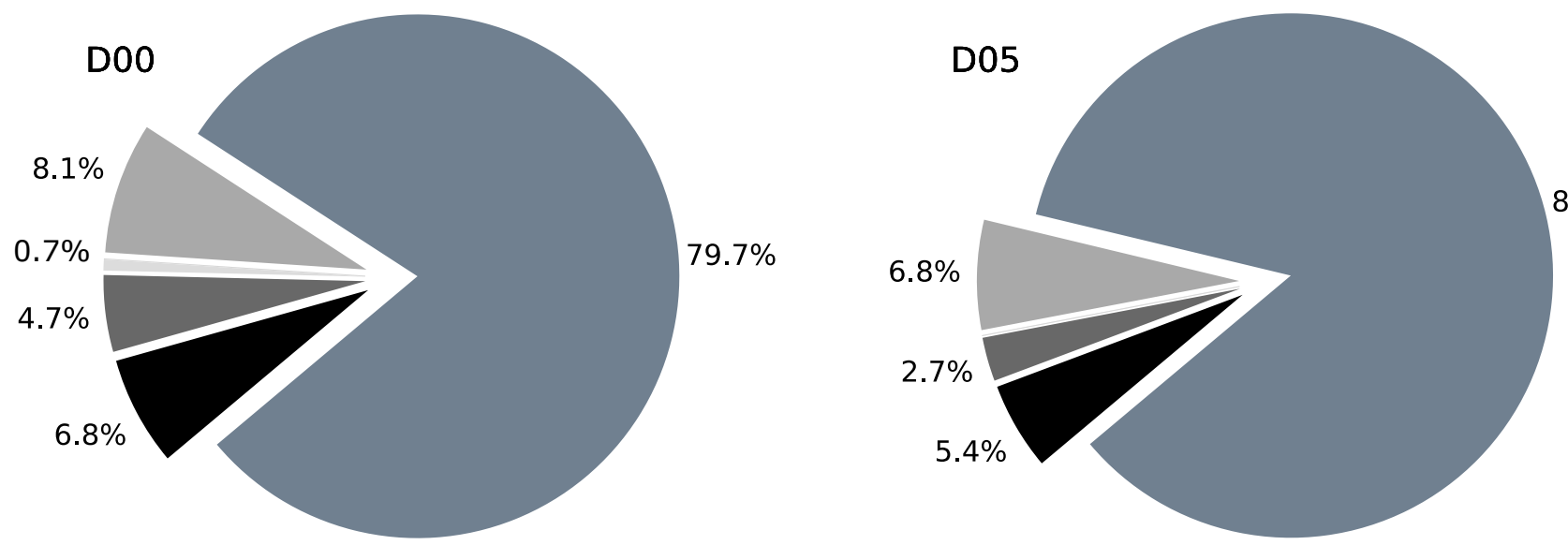

$85.1 \%$

Figure 4. The pie charts indicate the various evolutionary scenario of the BHB discussed in Sect. 3.1 and Sect. 3.2 for models C and D. The colour is referred to: the fraction of retained harder BHB $\left(h_{\text {ret }}\right)$, the fraction of ejected harder BHB $\left(h_{\text {esc }}\right)$, the fraction of escaped wider BHB $\left(w_{\text {ret }}\right)$, the fraction of retained wider BHB $\left(w_{\text {ret }}\right)$, the fraction of broke binaries retained $\left(b_{\text {ret }}\right)$, the fraction of broke binaries ejected $\left(b_{\text {esc }}\right)$ and the fraction of mergers (merge). On the other hand the striped slices referred to BHB that broke up. The width of each slice indicate the percentage as shown in Table 4 and Table 5.

contribute stripping stars from the cluster, accelerating its dissolution through the field. In our models the complete dissolution of the clusters occur between 1.5 Gyr and 3 Gyr.

We notice that the significant reduction of the BHB semi major axis (up to 1-2 order of magnitude) occurs in a time which ranges between $\sim 50$ and $\sim 7 \cdot 10^{2}$ Myr. In such time-range the clusters are still bound and the tidal forces have not yet contribute to dilute the clusters, avoiding the binary harden. The gravitational interactions that contribute to significantly shrink the BHB semi major axis act in a short time-range and in such time the cluster still contain between $60 \%$ and $80 \%$ of bound stars.

The complete disruption of clusters occur when the gravitational interactions do not play anymore a dynamical role in the evolution of the black hole binary. It is worth mentioning that such result are typical of open cluster that lie at $8.5 \mathrm{Kpc}$ from the Galactic center, otherwise clusters closer to the central regions would dissolve in a shorter time scale.

\section{SOURCES OF GRAVITATIONAL WAVES}

\subsection{Relativistic binaries}

The code that we used for this work (NBODY7) identifies those compact objects that will eventually merge due to the emission of gravitational radiation. Note that the NBODY7 code indicates a binary as 'merging' when at least one of the conditions described in Aarseth (2012) is satisfied ${ }^{1}$ (see also

\footnotetext{
${ }^{1}$ https://www.ast.cam.ac.uk/ sverre/web/pages/pubs.htm
} 
Table 5. Percentage of BHB mergers found for each model studied.

\begin{tabular}{cc}
\hline Model & \% \\
\hline A00 & 0.0 \\
A05 & 0.7 \\
\hline B00 & 0.7 \\
B05 & 0.7 \\
\hline C00 & 2.1 \\
C05 & 4.3 \\
\hline D00 & 7.1 \\
D05 & 5.7 \\
\hline
\end{tabular}

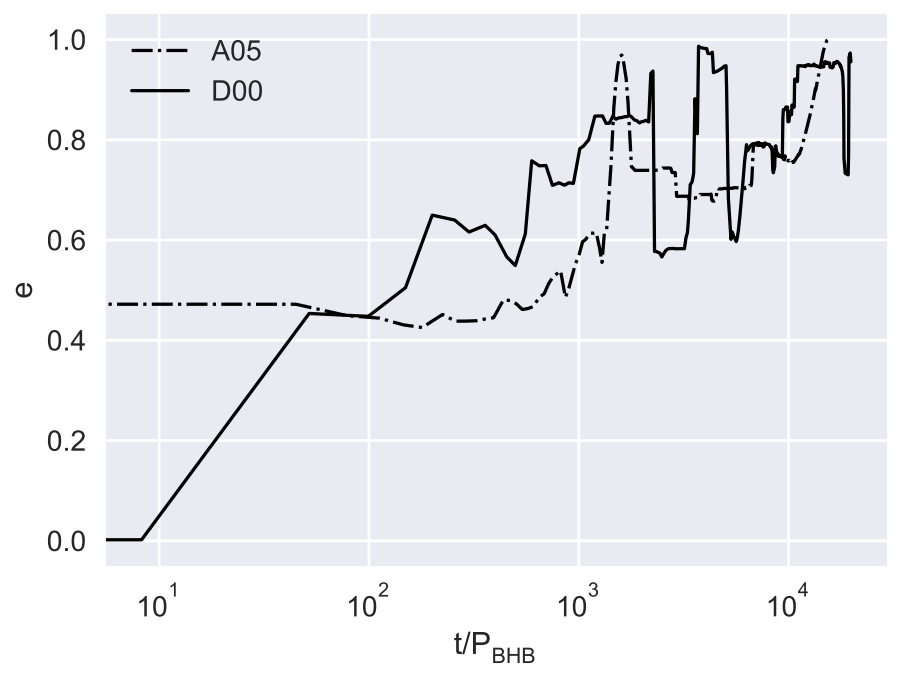

Figure 5. The evolution of the eccentricity for two cases in which the BHBs merge, for models A05 and D00.

Banerjee (2018a, Sect. 2.3.1)). However, the code does not integrate in time these binaries down to the actual coalescence, because this would require a reduction of the timestep down to such small values to make the integration stall. In Table 5 we give the percentage of BHB mergers as identified by NBODY7 in the simulations. The more massive the cluster, the larger the number of relativistic mergers found. We noted that the initial value of the binary eccentricity is not necessarily correlated with the number of coalescences. The majority of mergers occur in a time range between 5 Myr and 1.5 Gyr. Only two merger events take longer, between $\approx 1.5 \mathrm{Gyr}$ and $\approx 2$ Gyr. In our models, the clusters have not yet disrupted when the BHB coalescences occur, still containing more than the $80 \%$ of the initial number of stars.

In Figs. 6 and 7 we show the evolution of the BHB semimajor axis and pericentre distance of a few representative cases of Table 5 which initially were circular or eccentric, respectively. It is remarkable that the pericentre distances drop down to $7--8$ orders of magnitude with respect to the initial value. The eccentricities fluctuate significantly, episodically reaching values very close to unity.

Because of the relativistic recoil kick (Campanelli et al. 2007; Baker et al. 2006; González et al. 2007; Fragione et al. $2018 \mathrm{~b}, \mathrm{a})$, the product of the merger of the BHB might achieve very large velocities, such to escape the host cluster in all of the cases due to the very small escape velocity. Fig. 8 shows the distribution of the BHB semi-major axis and eccentricity in the last output before the gravitational wave regime drives the merger.

Because these binaries have undergone many dynamical interactions with other stars, the eccentricities are very high, ranging between 0.99996 and above 0.99999 .

Taking into account the expression for the GW emission time, $\mathcal{T}_{g w}$, (Peters 1964),

$\mathcal{T}_{g w}(y r)=5.810^{6} \frac{(1+q)^{2}}{q}\left(\frac{a}{10^{2} \mathrm{pc}}\right)^{4}\left(\frac{m_{1}+m_{2}}{10^{8} \mathrm{M}_{\odot}}\right)^{-3}\left(1-e^{2}\right)^{7 / 2}$

where $q$ is the mass ratio between the two BHs of mass $m_{1}$ and $m_{2}{ }^{2}$, we found that about $50 \%$ of the mergers are mediated by a three body encounter with a pertuber star.Such three body interaction is thus a fundamental ingredient for BHB coalescence in low dense star clusters, as already pointed out by Banerjee (2018a). An example of such mechanism is discussed in the next section (4.2).

\subsection{A detailed example of a merger event}

As we said above, NBODY7 identifies the binary merger events in a different way when a close interaction with a third object occurs.

However, it does not fully integrate those specific cases because following the detailed binary evolution would practically make the code stuck. So, in order to check with accuracy the process of BHB coalescence upon perturbation, we followed the evolution of one of the allegedly merging BHB by mean of the few-body integrator ARGdf (ArcaSedda \& Capuzzo-Dolcetta 2017a). Based on the ARCHAIN code (Mikkola \& Tanikawa 1999), ARGdf includes a treatment of dynamical friction effect in the algorithmic regularization scheme, which models at high precision strong gravitational encounters also in a post-Newtonian scheme with terms up to the 2.5 order (Mikkola \& Merritt 2008, whose first implementation in a direct-summation code is in Kupi et al. 2006). We chose, at random, one of our simulations of the D00 model to set initial conditions for the high precision evolution of a "pre merger" BHB considering its interaction with the closest 50 neighbours, number that we checked sufficient to give accurate predictions at regard.

This integration is a clear example of the relevance of dynamical interactions with other stars. Fig. 9 is a snapshot of the BHB evolution and the formation of a triple system with a pertuber star. ${ }^{3}$. The BHB shrinks by interacting with such pertuber, of mass $3.4 M_{\odot}$, which is in retrograde orbit as compared to the inner binary with an inclination of $105^{\circ}$ indicating an eccentric Kozai (1962) Lidov (1962) mechanism Naoz (2016). We note also a flyby star of mass $0.5 M_{\odot}$ which interacts with the triple system (BHB \& pertuber) In Fig. 10 we display the step-like increase of the BHB eccentricity, which is marked by the repeated interactions with the

2 note that the r.h.s of Eq. 2 is invariant on the choice $q=m_{1} / m_{2}$ or $q=m_{2} / m_{1}$

3 An animation of the triple orbit and the eccentricity evolution is available on line with the name triple_argdf.avi 

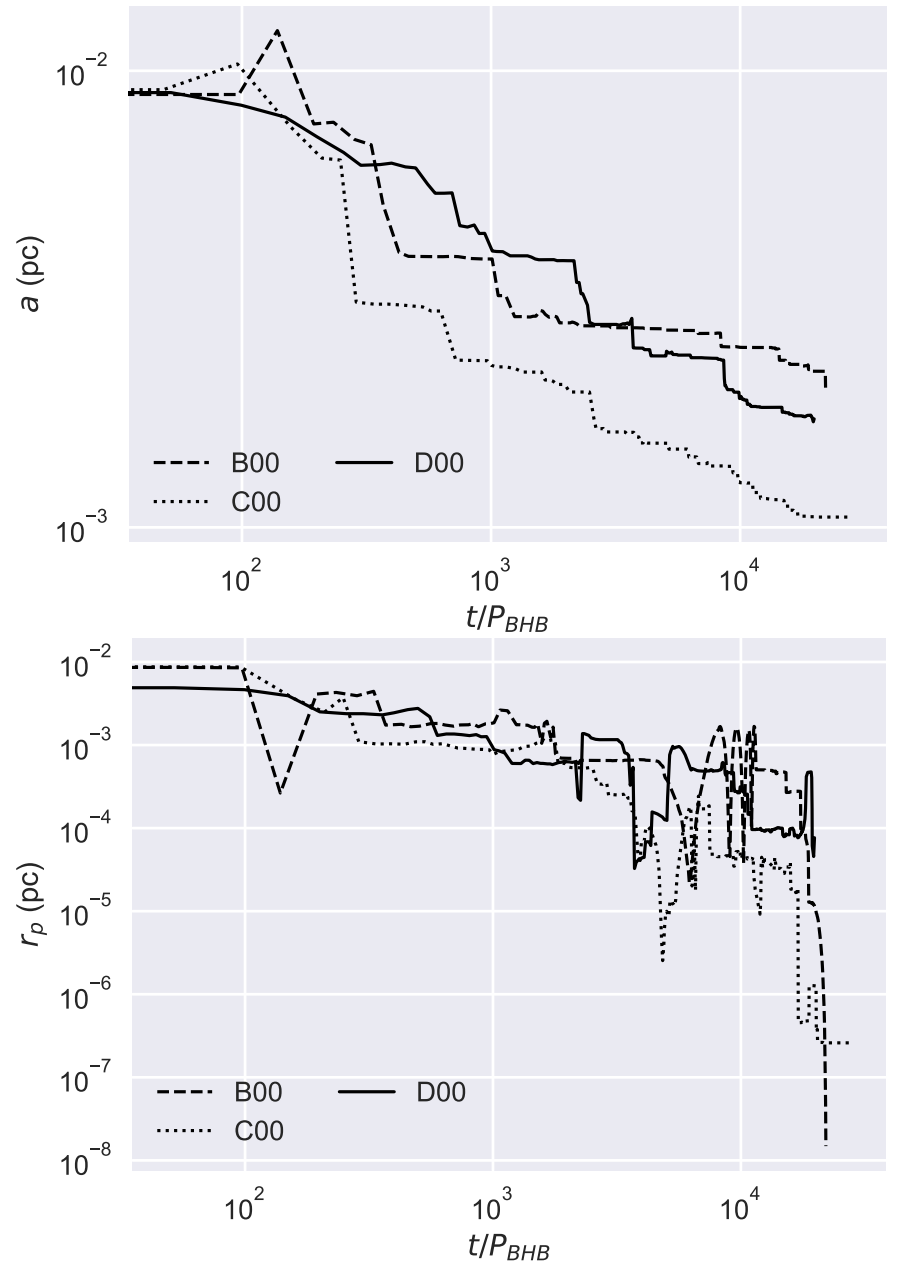

Figure 6. Evolution of the BHB semi major axis (upper panel) and pericentre distance (lower panel) of three illustrative cases which initially were circular.

outer star. Each time the pertuber orbits around the BHB we observe a step increasing of the eccentricity. On the contrary the flyby encounter is not efficient to make a significant perturbation on the eccentricity evolution. Fig. 11 shows a zoom of the evolution of the BHB latest orbits before the coalescence event. The plot in the rectangle is a zoom of the final part of the BHB trajectory (at its right side), spanning a length scale $\sim 10^{-7} \mathrm{pc}$. Therefore, in this particular case the triple built up is the main ingredient that drives the BHB coalescence. A similar result is derived by Banerjee (2018a) for low dense star clusters-like.

\subsection{Gravitational Waves}

In Fig. 12 we show the amplitude vs frequency of emitted gravitational waves for the case described in the above subsection. Using the last orbital parameters of the binary which correspond to the last integration made with ARGdf, we evolve the last phase of the binary by means of Eq.2 deriving a coalescence time $T_{\mathrm{mrg}} \cong 7 \mathrm{yrs}$. The amplitude is estimated following the approach of Keplerian orbits of Peters \& Mathews (1963) and the orbital evolution as in the work of Peters (1964). We have set the luminosity distance to that of the
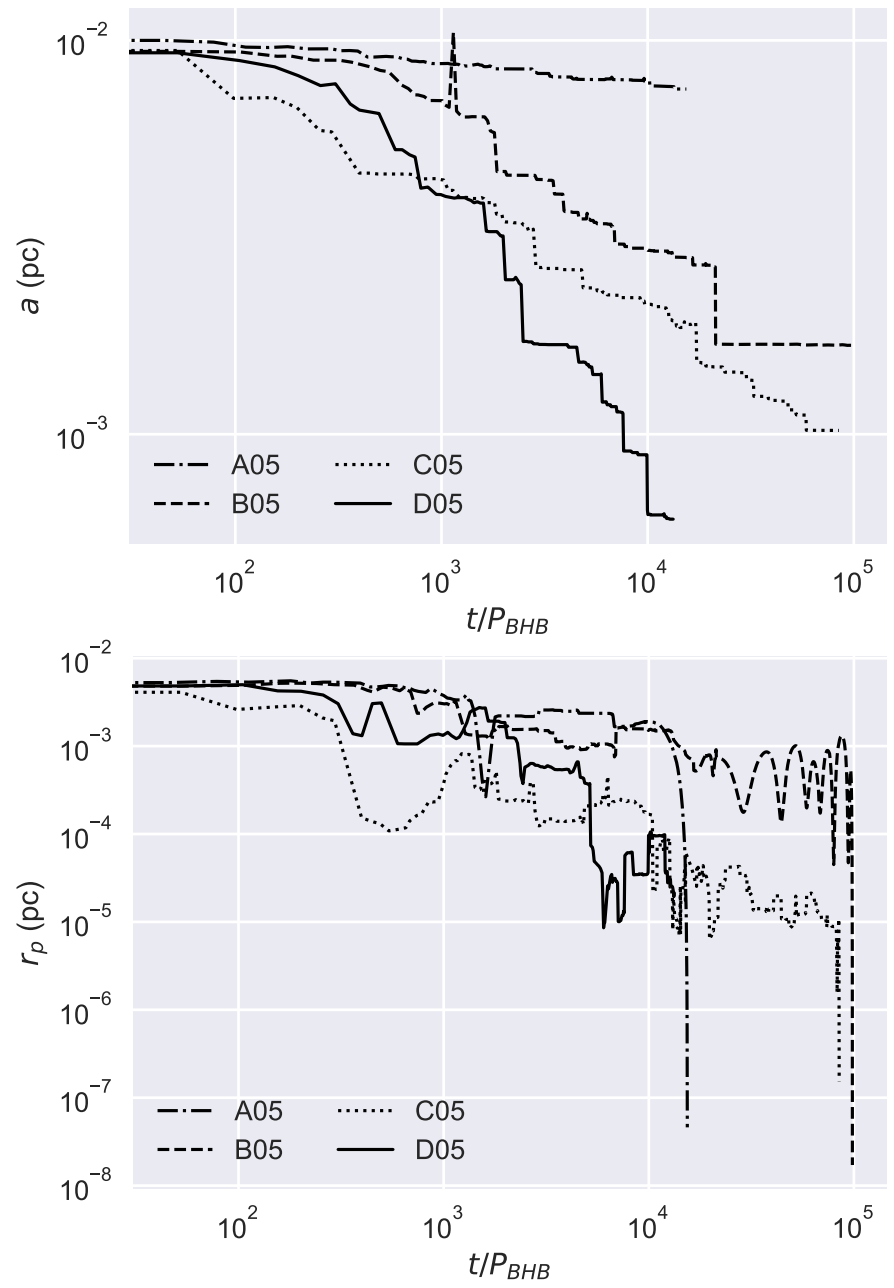

Figure 7. Same as Fig. 6 but for BHBs which initially had an eccentric orbit.

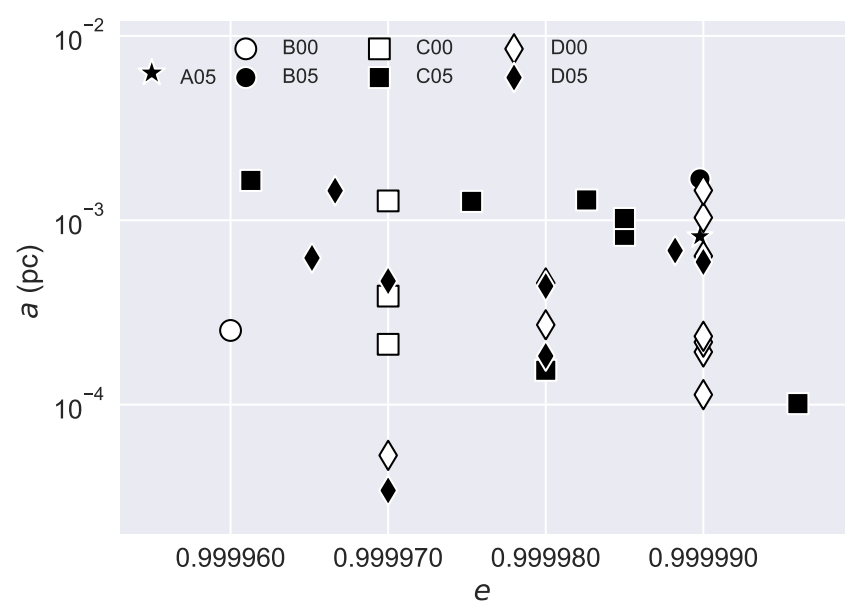

Figure 8. Distribution of the semi-major axis $(a)$ and eccentricity $(e)$ for all BHBs which merge in our simulations. The various symbols refer to the models as defined in Table 1. 


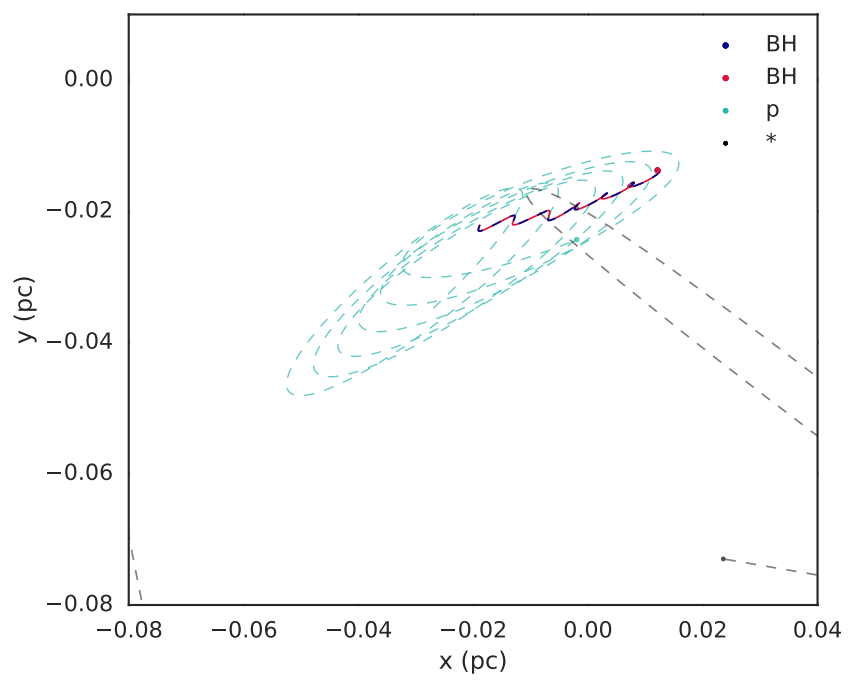

Figure 9. Trajectories of the BHs in our resimulation (model D00). The cyan circle and dashed line represents the perturbing star and its trajectory, the black holes are shown as a blue and red circle and solid lines. The grey circle and lines indicate the stars of the sub cluster sample simulated.

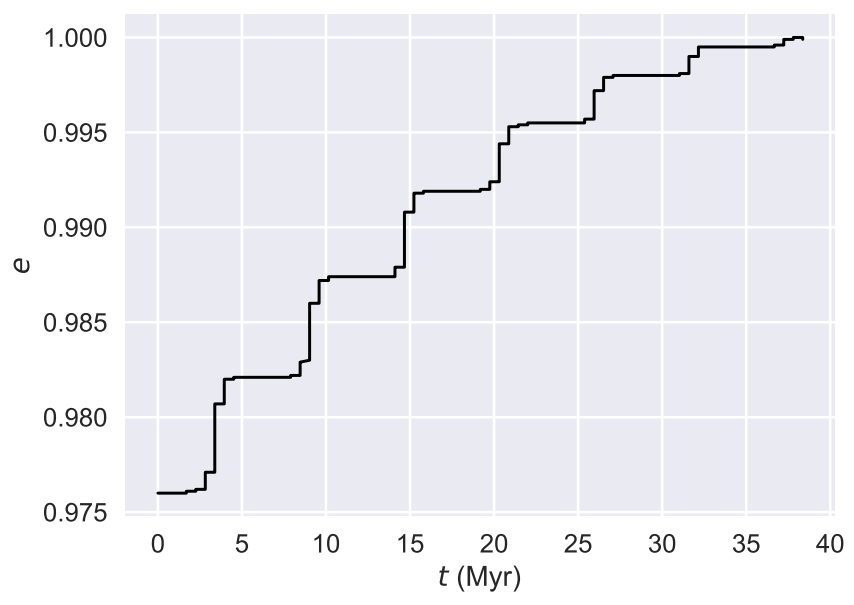

Figure 10. Evolution of the BHB eccentricity of Fig. 11 as a consequence of the three body encounter. Each jump in the eccentricity corresponds to a close passage of the third star to the $\mathrm{BHB}$, as described in the text.

first source detected by LIGO (Abbott et al. 2016a), which corresponds to a redshift of about $z=0.11$. As described by the work of Chen \& Amaro-Seoane (2017), only circular sources are audible by LISA, which is "deaf" to eccentric binaries of stellar-mass black holes that emit their maximum power at frequencies farther away from LISA. Hence, this particular source only enters the Advanced LIGO detection band.

\subsection{Black holes inspiraling outside of the cluster}

In our simulations some BHBs undergo a strong interaction with a star and they are kicked out from the cluster. The BHBs become escapers as defined in Section (3.2). In

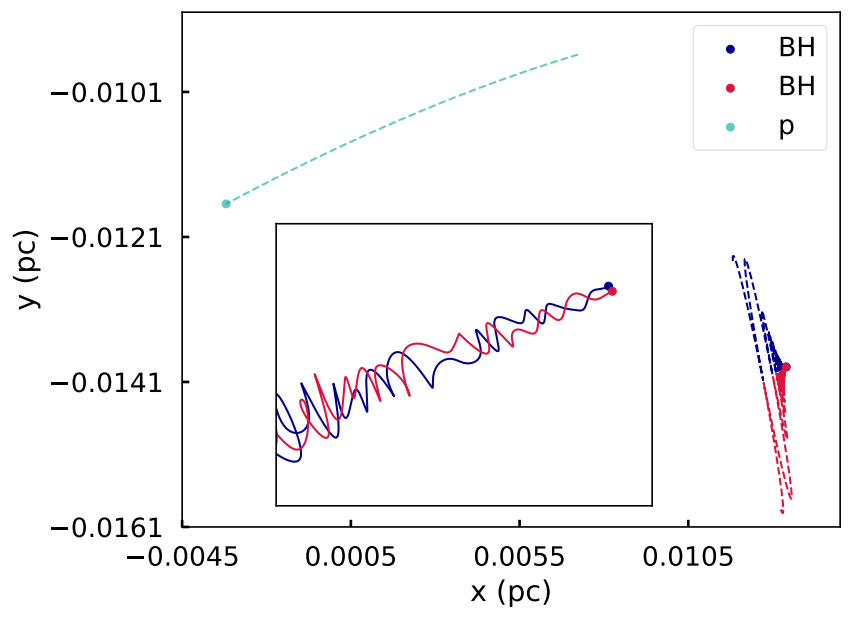

Figure 11. Trajectories of the BHs in our resimulation (model D00). The cyan circle and dashed line represents the perturbing star and its trajectory, the black holes are shown as a blue and red circle and solid lines.

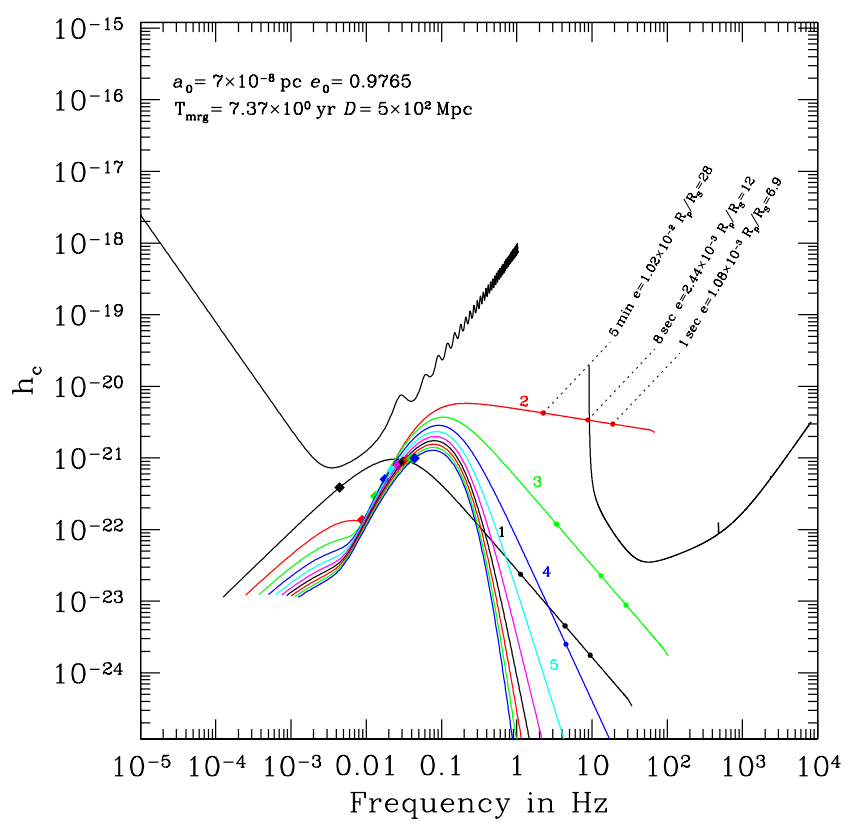

Figure 12. Characteristic amplitude $h_{c}$ of the first most important harmonics for the model of Fig. 11 at a luminosity distance of $D=500 \mathrm{Mpc}$. We also pinpoint seven moments in the evolution with dots which correspond, respectively, to $5 \mathrm{~min}, 8 \mathrm{sec}$ and 1 sec before the merger of the two black holes.

this case, the BHBs remain almost frozen in their relative configuration without any possible further evolution of their orbital parameters as described in Section (4.1): the escaping BHB evolves only due to the emission of gravitational radiation. For all these escaping BHBs (47 cases over the whole set of our simulations), we estimate the timescale for coalescence using the approach of Keplerian orbits of Peters (1964) and find that it always exceeds the Hubble time.

The inspiral phase of these binaries falls in the sensitivity window of LISA. However, they evolve very slowly in 
frequency due to the fact that the semi-major axis is still large, and the time to coalescence scales as $\propto a^{4}$. For an observational time of 5 years, the source would virtually not have moved in frequency, and hence the accumulated SNR over that time is negligible.

\subsection{Merger Rate}

To estimate approximately the merger rate, $\mathcal{R}_{\mathrm{mrg}}$ we first derive the mean number density of open clusters, $n_{\mathrm{OC}}$, over a volume $\Omega$ corresponding to redshift $z \leq 1$ as

$n_{\mathrm{OC}}=\frac{N_{\mathrm{OC}-\mathrm{MW}} N_{\mathrm{MW}-\Omega}}{\Omega}$.

In this equation $N_{\mathrm{OC}-\mathrm{MW}}$ is the number of OCs in Milky Way (MW)-like galaxies and $N_{\mathrm{MW}-\Omega}$ is the number of MWlike galaxies within $z=1$. We estimate the number of OCs in our Galaxy on the basis of the open-cluster mass function discussed in Piskunov et al. (2008); Portegies Zwart et al. (2010) for the mass range of OCs considered in our work (from $300 \mathrm{M}_{\odot}$ to approximately $3000 \mathrm{M}_{\odot}$ ). We take $\mathrm{N}_{\mathrm{MW}}=$ $10^{8}$ as the number of Milky Way-like galaxies at redshift $\sim 1$, as discussed in Alexander (2017). We stress here that the estimated merger rate is an upper limit, since it assumes that each open cluster host a massive BHB similarly to the clusters studied in our models.

Hence, the black hole binary meger rate can be estimated to be

$\mathcal{R}_{\mathrm{mrg}}=\frac{1}{N_{\mathrm{s}}} \sum_{k=1}^{N_{\mathrm{s}}} \frac{n_{\mathrm{OC}}}{t_{\mathrm{k}}} \approx 2 \mathrm{Gpc}^{-3} \mathrm{yr}^{-1}$,

where $\mathrm{N}_{\mathrm{S}}$ is the total number of $N$-body simulations performed in this work, and $t_{k}$ is the time of each coalescence event as found in our simulations. This estimate is however derived under the most favourable conditions and represents the most optimistic merger rate expected from low-mass open clusters. Note that the BHB merger rate inferred from the first LIGO observations (GW150914) is in the range 2 - $600 \mathrm{Gpc}^{-3} \mathrm{yr}^{-1}$ (Abbott et al. 2016c). The most updated estimate of the merger rate from LIGO-Virgo events (after including GW170104) is 12-213 $\mathrm{Gpc}^{-3} \mathrm{yr}^{-1}$ (Abbott et al. 2017a). Our BHB merger rate is consistent with those found in Banerjee (2017, 2018b,a) for BHB mergers in Young Massive Star Clusters (YMSC). Antonini \& Rasio (2016) found a merger rate ranging from 0.05 to $1 \mathrm{Gpc}^{-3} \mathrm{yr}^{-1}$ for possible progenitor of GW150914 in globular clusters at $\mathrm{z}<0.3$. Rodriguez et al. (2016b) and Rodriguez et al. (2016a) derived that in the local universe BHBs formed in Globular Clusters (GCs) will merge at a rate of $5 \mathrm{Gpc}^{-3} \mathrm{yr}^{-1}$. A result very similar was derived by Askar et al. (2017) who, for BHB originated in globular cluster, derived a rate of $5.4 \mathrm{Gpc}^{-3}$ $\mathrm{yr}^{-1}$. When the history of star clusters across cosmic time is included, Fragione \& Kocsis (2018) showed that the rate in the local Universe is $\sim 10 \mathrm{Gpc}^{-3}$, i.e. nearly twice the rate predicted for isolated clusters.

In Fig. 13 we show the estimated merger rate as a function of the initial number of cluster stars $(N)$. The merger rates derived from our models $\mathrm{A}, \mathrm{B}, \mathrm{C}$ and $\mathrm{D}$ are well fitted with a linear relation. An extension of our merger rate estimate to globular cluster-like systems $\left(N>10^{5}\right)$ gives a

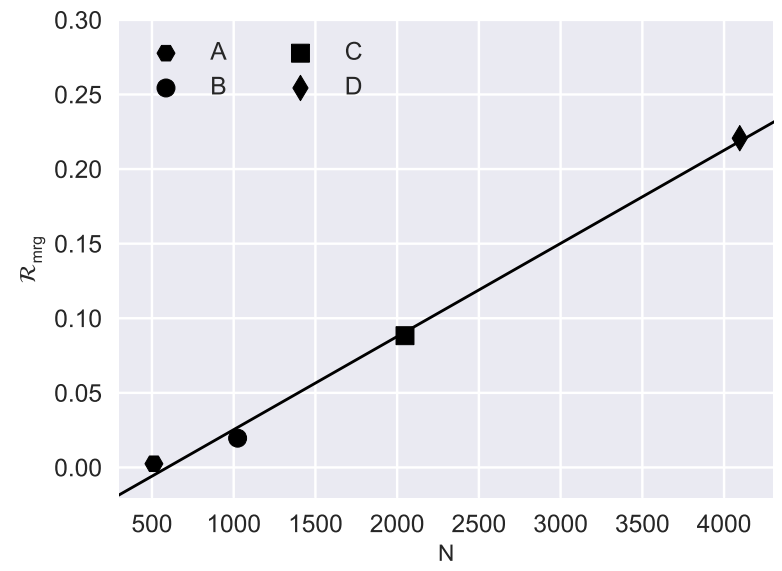

Figure 13. The most optimistic merger rate $\left(\mathcal{R}_{\mathrm{mrg}}\right.$, in $\mathrm{Gpc}^{-3}$ $\mathrm{yr}^{-1}$ ) obtained for each model studied in this work, as function of the total initial number of cluster stars $N$. The merger rates are well fitted with a linear relation, $R_{\mathrm{mrg}}=a N+b$, where $a=6.2 e-05$ and $b=-0.04$.

result in agreement with that found in Park et al. (2017), and previously found by Bae et al. (2014) and Rodriguez et al. (2016a,b).

Although BHB mergers originating in open clusters-like systems might be less numerous than those produced in massive star clusters, they would add a comparable amount to the BHB merger rate in the Universe because of their larger abundance (Banerjee 2018b,a).

\section{TIDAL DISRUPTION EVENTS AND BHB EJECTION}

All numerical models we have considered so far have solar metallicity, $Z=2.02$, and are based on the standard stellar evolution recipes (Hurley et al. 2000, 2002).

Moreover, they all consider an equal-mass BHB sitting in the host cluster centre with an initial mass $M_{\mathrm{BHB}}=60$ $\mathrm{M}_{\odot}$. In order to explore the role played by metallicity, stellar evolution recipes adopted, BHB mass and mass ratio, we present in this section an additional sample consisting of 421 simulations (the supplementary models), gathered in 5 different groups.

In all these 5 supplementary groups, the OC initial conditions are the same as in D00 principal models. This implies $N_{\mathrm{cl}}=4096$ and, for the BHB initial orbit, $a_{\mathrm{BHB}}=0.01 \mathrm{pc}$ and $e_{\mathrm{BHB}}=0$, unless specified otherwise. We labels each group with the letter $\mathrm{M}$ and a number between 1 and 5 .

In model M1, we model the OC assuming an initial metallicity value $Z=0.0004$, typical of an old stellar population. The BHB initial conditions are the same as in model D00. Stellar evolution is treated taking advantage of a new implementation of the SSE and BSE tools, which includes metal-dependent stellar winds formulae and improvements described in Belczynski et al. (2010d). In the following, we identify the updated stellar evolution treatment used for model M1 as BSEB, while in all the other cases we label 
Table 6. Supplementary models. The columns refer to: model name, BHB individual masses and mass ratio, metallicity, stellar evolution recipes used, number of simulations performed. The cluster is always simulated with 4096 stars.

\begin{tabular}{ccccccc}
\hline Model & $\begin{array}{c}M_{1} \\
\mathrm{M}_{\odot}\end{array}$ & $\begin{array}{c}M_{2} \\
\mathrm{M}_{\odot}\end{array}$ & $\boldsymbol{q}$ & $\begin{array}{c}\boldsymbol{Z} \\
\mathrm{Z}_{\odot}\end{array}$ & $\mathrm{SE}$ & $N_{\text {mod }}$ \\
\hline M1 & 30 & 30 & 1 & $10^{-4}$ & BSEB & 109 \\
M2 & 30 & 30 & 1 & $10^{-4}$ & BSE & 131 \\
M3 & 13 & 7 & 0.54 & 1 & BSE & 100 \\
M4 & 30 & 7 & 0.23 & 1 & BSE & 42 \\
M5 & 30 & 30 & 1 & 1 & BSE & 89 \\
\hline
\end{tabular}

them with BSE. Note that these updates allow the formation of BHs with natal masses above $30 \mathrm{M}_{\odot}$, while this is not possible in the standard SSE implementation (Hurley et al. 2000). Moreover, it must be stressed that the updates affect only metallicities below the solar value.

Model M2 is similar to model M1 in terms of initial metallicity and $\mathrm{BHB}$ initial condition, while we used the standard SSE and BSE codes to model stellar evolution. Therefore, the underlying difference between this and the previous is that in the latter the mass of compact remnants is systematically lower. This, in turn, implies that the number of perturbers that can have a potentially disruptive effect on the BHB evolution is reduced in model M2.

In model M3 we adopt $Z=0.02$, i.e. solar values, and we focuse on a BHB with component masses $M_{1}=13 \mathrm{M}_{\odot}$ and $M_{2}=7 \mathrm{M}_{\odot}$. This set has a twofold focus. On one side, it allows us to investigate the evolution of a $\mathrm{BHB}$ with mass ratio lower than 1 . On the other side, since in this case the BHB total mass is comparable to the maximum mass of compact remnants allowed from stellar evolution recipes, gravitational encounters should be more important in the BHB evolution.

To further investigate the role of mass ratio, M4 models are similar to $\mathrm{M} 3$, but in this case the BHB mass ratio is smaller, namely $q=0.23$, i.e. the components mass are $M_{1}=$ $30 \mathrm{M}_{\odot}$ and $M_{2}=7 \mathrm{M}_{\odot}$.

In all the principal and supplementary models discussed above, we assume that the BHB is initially at the centre of the OC. In order to investigate whether such a system can be formed dynamically, i.e. via strong encounters, in model M5 we set two BHs, with masses $M_{1}=M_{2}=30 \mathrm{M}_{\odot}$, initially unbound. In this case we set $Z=0.02$, in order to compare with D00 principal models.

The results of these runs are summarized in Table 7.

Since we are interested only in the evolution of the initial BHB, we stop the simulations if at least one of the BHB initial components is ejected away from the parent OC.

When metallicity-dependent stellar winds are taken into account (model M1), the reduced mass loss causes the formation of heavier compact remnants, with masses comparable to the BHB components. Since the number of BHs is $\sim 10^{-3} N_{\mathrm{cl}}$, according to a Kroupa IMF, in models M1 at least 4-5 heavy BHs can form, interact and possibly disrupt the initial BHB. This is confirmed in the simulations results - we find one of the BHB components kicked out in $P_{\text {esc }}=34.9 \%$ of the cases investigated. After the component ejection, the remaining $\mathrm{BH}$ can form a new binary with one of the perturbers, or a new BHB.
The "ejection probability" in models M2 is only slightly lower than in M1, $P_{\text {esc }}=33.6 \%$, thus implying that the heavier perturbers forming in models M1 only marginally affect the BHB evolution. This is likely due to two factors: (i) their number is relatively low (4-5), (ii) the mass segregation process in such a low-density, relatively light stellar system is slower than the time over which stellar encounters determine the BHB evolution. The latter point implies that the $\mathrm{BHB}$ evolution is mostly driven by the cumulative effects of multiple stellar encounters, rather than to a few interactions with a heavy perturber.

In model M3, characterized by a lighter BHB and solar metallicity, the BHB total mass falls in the high-end of the $\mathrm{BH}$ mass spectrum, $20 \mathrm{M}_{\odot}$. This implies a larger number of massive perturbers with respect to the standard case discussed in the previous sections and provides insight on the fate of light BHBs in OCs. Due to the high-efficiency of strong interactions, the BHB unbinds in $f_{\mathrm{esc}}=32 \%$ of the cases, and in no case the BHB undergoes coalescence.

Model M5 is characterized by a similar ejection probability, which instead rises up to $40.5 \%$ in model M4. This is likely due to the relatively low-mass of the secondary component. Indeed, as shown through scattering experiments, BHB-BH interactions seem to naturally lead to a final state in which the resulting $\mathrm{BHB}$ has a larger mass ratio (see for instance Arca-Sedda et al. 2018).

In a few cases, we found that the BHB disruption is mediated by a star, which binds to one of the two BHB former components. The newly formed BH-star pair is characterized by a high eccentricity $(e>0.9)$ and pericentre sufficiently small to rip the star apart and give rise to a tidal disruption event (TDE). In the current Nbody6 implementation, only the $10 \%$ of the star mass is accreted on the $\mathrm{BH}$, while this percentage can be as high as $50 \%$.

The fraction of models in which a TDE takes place spans one order of magnitude, being $f_{\mathrm{TDE}} \cong 0.03-0.3$, with the maximum achieved in models M4 and the minimum in M1. Note that in model M5 we did not found any TDE (see Table 7 ), but in this case the two BHs are initially moving outside the $\mathrm{OC}$ inner regions.

In our models, TDEs involve either main sequence stars (MS), stars in the core He burning phase (HB) or in the early asymptotic giant branch (AGB) phase. In model M3 $\left(f_{\mathrm{TDE}}=0.14\right)$ TDEs involve MS $(29 \%)$, early $\mathrm{AGB}(57 \%)$ and AGB (14\%) stars. In model M4, where the BHB has a low mass ratio $(q=7 / 30)$, TDEs are boosted, since in this case is easier to replace the lighter $\mathrm{BH}$. Indeed, a component swap occurs in $28.5 \%$ of the cases, with the new companion star being swallowed by the heavier BH.

Our findings suggest that X-ray or UV emission from OCs can be the signature of the presence of BHs with masses as high as $20-30 \mathrm{M}_{\odot}$.

Using our results we can calculate the TDE rate for Milky Way - like galaxies as

$\Gamma_{\mathrm{TDE}}=\frac{f_{\mathrm{TDE}} N_{\mathrm{OC}} N_{\mathrm{MW}}}{\Omega T}=0.3-3.07 \times 10^{-6} \mathrm{yr}^{-1}$,

Our estimates nicely agree with similar TDE-rate calculation provided by Perets et al. (2016), and results in a $\sim 1$ order of magnitude lower than the values calculated for TDEs occurring around supermassive black holes (Fragione \& Leigh 2018; Stone \& Metzger 2016; Stone et al. 2017; 
Table 7. Summary of results from the supplementary models. Columns refer to: model name, percentage of cases in which at least one of the BHB components is ejected, percentage of cases in which a star is swallowed by one of the two BHs, percentage of cases in which the BHB merges.

\begin{tabular}{cccc}
\hline Model & $\begin{array}{c}P_{\text {esc }} \\
\%\end{array}$ & $\begin{array}{c}P_{\text {TDE }} \\
\%\end{array}$ & $\begin{array}{c}P_{\text {mer }} \\
\%\end{array}$ \\
\hline M1 & 34.9 & 2.8 & 0.0 \\
M2 & 33.6 & 6.9 & 3.8 \\
M3 & 32.0 & 14.0 & 0.0 \\
M4 & 40.5 & 28.5 & 0.0 \\
M5 & 32.6 & 0.0 & 0.0 \\
\hline
\end{tabular}

Stone \& van Velzen 2016; Arca-Sedda \& Capuzzo-Dolcetta $2017 \mathrm{~b})$. Here $f_{\text {TDE }}$ is the fraction of TDE inferred from simulation, while we adopt the values for $N_{\mathrm{OC}}, N_{\mathrm{MW}}$ and $\Omega$ discussed in the previous section. Moreover, we assumed $T=3$ Gyr, i.e. the simulated time.

We apply the same analysis to our principal models and find a TDE rate for solar-metallicity OCs of $\Gamma_{\mathrm{TDE}}=0.3-$ $3.07 \times 10^{-6} \mathrm{yr}^{-1}$ for MW-like galaxies in the local Universe.

The BHB coalescence occurs in a few cases $f_{\text {mer }} \cong 0.004$, and only in models M2, where metallicity dependent mass loss is disabled. This suggests that there exists three different regimes, depending on the perturber maximum mass $M_{p}$. If (1) $M_{\mathrm{BHB}} \gg M_{p}$, the BHB is much more massive than field stars and stellar encounters poorly affects its evolution; however, if (2) $M_{\mathrm{BHB}} \geq M_{p}$, a few perturbers have masses comparable to the $\mathrm{BHB}$, and can efficiently drive it toward coalescence, causing for instance an increase in the BHB eccentricity or a considerable shrinkage; in case (3) that $M_{\mathrm{BHB}}=M_{p}$, there is at least one perturber with a mass similar, or even larger, than the BHB. The BHB-perturber interactions causes either the BHB disruption, or the formation of a new BHB with the perturber replacing the lighter BHB component.

Note that we cannot exclude that a BHB merge in other models, since we stop the computation if the original $\mathrm{BHB}$ gets disrupted. Hence, we can infer a lower merger rate for metal poor OCs as follows

$\mathcal{R}_{\mathrm{mrg}}=\frac{f_{\mathrm{mer}} N_{\mathrm{MW}} N_{\mathrm{OC}}}{\Omega T} \simeq 0.26 \mathrm{yr}^{-1} \mathrm{Gpc}^{-3}$.

These models highlight the importance of stellar evolution in our calculations, since stronger stellar winds lead to smaller remnants reducing the number of objects massive enough to cause the BHB disruption. This leads to a higher probability for the BHB to shrink by the repeated interactions with smaller objects.

As described above, in model M5 the two BHs are initially unbound, and their initial position and velocities are kept coherently to the OC distribution function. In this situation, the fraction of cases in which at least one of the $\mathrm{BHs}$ is ejected from the cluster is similar to that of the other models $\left(f_{\text {esc }} \sim 32.6 \%\right)$, but in none of the models the two BHs bind together. This is due to the low efficiency of dynamical friction in the OC that avoids the two BHB to decay in the innermost potential well. Also TDEs are suppressed, due to the low number of strong encounters between $\mathrm{BH}$ and cluster stars because of the low density of the surrounding environment.

To conclude, our supplementary models confirm that the possibility for a BHB to coalesce in an OC depends strongly on the environment in which the BHB formed and on its total mass and mass ratio. In metal-poor OCs (metaldependent) stellar winds drive the formation of a seizable number of massive perturbers that can efficiently disrupt the $\mathrm{BHB}$, thus reducing the coalescence probability. Coalescence is strongly reduced also in the case of low mass ratios $(q \sim 0.2)$ or relatively light BHBs $\left(M_{1}+M_{2} \sim 20 \mathrm{M}_{\odot}\right)$.

One of the most interesting outcomes of the models presented in this section is the possibility to use the OC TDE rate as a proxy to infer the presence of a massive $\mathrm{BH}$ or $\mathrm{BHB}$ around the OC centre.

\section{CONCLUSIONS}

In this paper we address the evolution of an equal mass, nonspinning, stellar BHB with total mass $60 \mathrm{M}_{\odot}$ inhabiting the centre of a small/intermediate star cluster (open cluster like, OC), using the direct N-body code NBODY7(Aarseth 2012). In order to quantify the effect of repeated stellar encounters on the BHB evolution, we vary the OC total mass and the BHB orbital properties, providing a total of $\sim 1150$ simulations which we refer to as principal models. For the sake of comparison, we also investigate the role played by the BHB total mass, the stellar evolution recipes adopted and the OC metallicity. These can be considered as supplementary models. The total simulations sample hence consists of $\sim 1500$ different OC models, with masses in the range $300-3000$ $\mathrm{M}_{\odot}$.

In $\sim 95 \%$ of all the principal simulations performed, the BHB hardens due to the repeated scatterings with flyby stars, while its eccentricity increases significantly. This process takes place on a relatively short time-scale, $\sim 1$ Gyr. In $\sim 1.2 \%$ of the principal simulations, instead, the perturbations induced by massive stars that occasionally approach the BHB make it wider. In the remaining $\sim 4.8 \%$ cases, the interactions with OC stars are sufficiently strong to break up the BHB. When the BHB gets harder, its semi-major axis reduces by 2 to 4 orders of magnitude, thus decreasing the merger time-scale by a factor 16 in the best case. Hardened BHBs are retained within the parent $\mathrm{OC}$ with a probability of $95 \%$, while those becoming wider are all retained. In the case of BHB breakup, the two BHs tend to form short-lived binary systems with other OC stars, and eventually at least one of the two BHs is ejected from the parent cluster.

In $\sim 3 \%$ of the models, the star-BHB interactions are sufficiently effective to drive the BHB coalescence within a Hubble time. We find that a crucial ingredient for the BHB to merge is the interaction with a perturbing star, which considerably shortens the merger time. These dynamical perturbers enhance the number of GW sources by as much as $50 \%$. The merger takes place in a time ranging from $5 \mathrm{Myr}$ to 2.9 Gyr. In a few cases, the merging binaries emit GWs shifting from the $10^{-3}$ to the $10 \mathrm{~Hz}$ frequency band. This suggests that merging BHBs in OCs can potentially be seen both by LISA, $200 \mathrm{yr}$ before the merger, and LIGO, during the last phase preceding the merger.

Extrapolating our results to the typical population of 
OCs in MW-like galaxies in the local Universe, we found that the most optimistic merger rate for BHB mergers in low-mass stellar systems is $\mathcal{R}_{\mathrm{mrg}} \sim 2 \mathrm{yr}^{-1} \mathrm{Gpc}^{-3}$, a value compatible with the merger rate expected for galactic nuclei, but smaller than the merger rate inferred for globular and young massive clusters.

According to our supplementary models, in low-metal environments the BHB hardening is suppressed, due to the presence of a large number of high-mass perturbers that can efficiently drive the BHB disruption. In this regard, different stellar evolution recipes may affect significantly the results, since they regulate the maximum mass of compact remnant. Assuming a smaller BHB and a solar metallicity for the cluster stars leads to similar results, since, again, the fraction of perturbers sufficiently massive to drive the BHB disruption is much larger.

In none of the cases in which the BHB components are initially kept unbound the BHB forms via dynamical processes. This is due to the low efficiency of dynamical friction in the OC low-dense environment, which is unable to drive the $\mathrm{BHs}$ orbital segregation and pairing. So binaries as the ones considered in this paper should be primordial.

In a noticeable fraction of the supplementary models, we found that the BHB breaks up and one of the BHs forms a very eccentric binary with an OC star, typically a main sequence or an AGB star. These binaries are usually shortliving systems and result in a tidal disruption event, with part of the stellar debris being swallowed by the BH.

Our supplementary models suggest that TDEs in OCs occur at a rate $\Gamma_{\mathrm{TDE}}=3.08 \times 10^{-6} \mathrm{yr}^{-1}$ per MW-like galaxies in the local Universe.

\section{ACKNOWLEDGEMENTS}

SR acknowledges Sapienza, Universitá di Roma, which funded the research project "Black holes and Star clusters over mass and spatial scale" via the grant AR11715C7F89F177. SR is thankful to Sverre Aarseth of the Institute of Astronomy, Cambridge, for his helpful comments and suggestions during the development of this work. MAS acknowledges the Sonderforschungsbereich SFB 881 "The Milky Way System" (subproject Z2) of the German Research Foundation (DFG) for the financial support provided. PAS acknowledges support from the Ramón y Cajal Programme of the Ministry of Economy, Industry and Competitiveness of Spain, as well as the COST Action GWverse CA16104. GF is supported by the Foreign Postdoctoral Fellowship Program of the Israel Academy of Sciences and Humanities. GF also acknowledges support from an Arskin postdoctoral fellowship and Lady Davis Fellowship Trust at the Hebrew University of Jerusalem.

\section{REFERENCES}

Aarseth S. J., 1973, Vistas in Astronomy, 15, 13

Aarseth S. J., 2003, Gravitational N-Body Simulations

Aarseth S. J., 2012, MNRAS, 422, 841

Abbott B. P., et al., 2016a, Physical Review Letters, 116, 061102 Abbott B. P., et al., 2016b, Physical Review Letters, 116, 241103 Abbott B. P., et al., 2016c, ApJ, 833, L1

Abbott B. P., et al., 2017a, Physical Review Letters, 118, 221101
Abbott B. P., et al., 2017b, Physical Review Letters, 119, 141101 Abbott B. P., et al., 2017c, Physical Review Letters, 119, 161101 Alexander T., 2017, ARA\&A, 55, 17

Amaro-Seoane P., Chen X., 2016, MNRAS, 458, 3075

Antonini F., Rasio F. A., 2016, ApJ, 831, 187

Antonini F., Chatterjee S., Rodriguez C. L., Morscher M., Pattabiraman B., Kalogera V., Rasio F. A., 2016, ApJ, 816, 65

Arca-Sedda M., Capuzzo-Dolcetta R., 2017a, preprint, (arXiv:1709.05567)

Arca-Sedda M., Capuzzo-Dolcetta R., 2017b, MNRAS, 471, 478

Arca-Sedda M., Capuzzo-Dolcetta R., Antonini F., Seth A., 2015, ApJ, 806, 220

Arca-Sedda M., Li G., Kocsis B., 2018, preprint, (arXiv: 1805.06458)

Askar A., Szkudlarek M., Gondek-Rosińska D., Giersz M., Bulik T., 2017, MNRAS, 464, L36

Bae Y.-B., Kim C., Lee H. M., 2014, MNRAS, 440, 2714

Baker J. G., Centrella J., Choi D.-I., Koppitz M., van Meter J. R., Miller M. C., 2006, ApJ, 653, L93

Banerjee S., 2017, MNRAS, 467, 524

Banerjee S., 2018a, MNRAS,

Banerjee S., 2018b, MNRAS, 473, 909

Banerjee S., Baumgardt H., Kroupa P., 2010, MNRAS, 402, 371

Belczynski K., Kalogera V., Bulik T., 2002, ApJ, 572, 407

Belczynski K., Bulik T., Fryer C. L., Ruiter A., Valsecchi F., Vink J. S., Hurley J. R., 2010a, ApJ, 714, 1217

Belczynski K., Bulik T., Fryer C. L., Ruiter A., Valsecchi F., Vink J. S., Hurley J. R., 2010b, ApJ, 714, 1217

Belczynski K., Bulik T., Fryer C. L., Ruiter A., Valsecchi F., Vink J. S., Hurley J. R., 2010c, ApJ, 714, 1217

Belczynski K., Bulik T., Fryer C. L., Ruiter A., Valsecchi F., Vink J. S., Hurley J. R., 2010d, ApJ, 714, 1217

Benacquista M. J., Downing J. M. B., 2013, Living Reviews in Relativity, 16, 4

Bethe H. A., Brown G. E., 1998, ApJ, 506, 780

Binney J., Tremaine S., 2008, Galactic Dynamics: Second Edition. Princeton University Press

Campanelli M., Lousto C., Zlochower Y., Merritt D., 2007, ApJ, 659 , L5

Chen X., Amaro-Seoane P., 2017, ApJ, 842, L2

Dehnen W., 1993, MNRAS, 265, 250

Downing J. M. B., Benacquista M. J., Giersz M., Spurzem R., 2010, MNRAS, 407, 1946

Duquennoy A., Mayor M., 1991, A\&A, 248, 485

Fragione G., Kocsis B., 2018, preprint, (arXiv:1806.02351)

Fragione G., Leigh N., 2018, MNRAS, 479, 3181

Fragione G., Leigh N., Ginsburg I., Kocsis B., 2018a, arXiv:1806.08385,

Fragione G., Ginsburg I., Kocsis B., 2018b, ApJ, 856, 92

Fregeau J. M., Cheung P., Portegies Zwart S. F., Rasio F. A., 2004, MNRAS, 352, 1

Fryer C. L., Belczynski K., Wiktorowicz G., Dominik M., Kalogera V., Holz D. E., 2012, ApJ, 749, 91

Giacobbo N., Mapelli M., 2018, MNRAS, 480, 2011

González J. A., Sperhake U., Brügmann B., Hannam M., Husa S., 2007, Physical Review Letters, 98, 091101

Goswami S., Kiel P., Rasio F. A., 2014, ApJ, 781, 81

Heger A., Fryer C. L., Woosley S. E., Langer N., Hartmann D. H., 2003, ApJ, 591, 288

Heggie D. C., 1975, MNRAS, 173, 729

Hills J. G., 1975, Nature, 254, 295

Hurley J. R., Pols O. R., Tout C. A., 2000, MNRAS, 315, 543

Hurley J. R., Tout C. A., Pols O. R., 2002, MNRAS, 329, 897

Janka H.-T., 2013, MNRAS, 434, 1355

Kimpson T. O., Spera M., Mapelli M., Ziosi B. M., 2016, MNRAS, 463,2443

Kozai Y., 1962, AJ, 67, 591

Kroupa P., 2001, MNRAS, 322, 231 
Kupi G., Amaro-Seoane P., Spurzem R., 2006, doi:10.1111/j.17453933.2006.00205.x, pp L77+

Lidov M. L., 1962, Planet. Space Sci., 9, 719

Loeb A., 2016, ApJ, 819, L21

Mapelli M., 2016, MNRAS, 459, 3432

Mapelli M., Bressan A., 2013, MNRAS, 430, 3120

Mapelli M., Moore B., Giordano L., Mayer L., Colpi M., Ripamonti E., Callegari S., 2008, MNRAS, 383, 230

Mapelli M., Huwyler C., Mayer L., Jetzer P., Vecchio A., 2010, ApJ, 719, 987

Mathieu R. D., 2008, in Vesperini E., Giersz M., Sills A., eds, IAU Symposium Vol. 246, Dynamical Evolution of Dense Stellar Systems. pp 79-88, doi:10.1017/S1743921308015366

Mikkola S., Merritt D., 2008, AJ, 135, 2398

Mikkola S., Tanikawa K., 1999, MNRAS, 310, 745

Miyamoto M., Nagai R., 1975, PASJ, 27, 533

Naoz S., 2016, ARA\&A, 54, 441

Park D., Kim C., Lee H. M., Bae Y.-B., Belczynski K., 2017, MNRAS, 469, 4665

Perets H. B., Li Z., Lombardi Jr. J. C., Milcarek Jr. S. R., 2016, ApJ, 823, 113

Perna R., Chruslinska M., Corsi A., Belczynski K., 2018, MNRAS, 477,4228

Peters P. C., 1964, Phys. Rev., 136, B1224

Peters P. C., Mathews J., 1963, Physical Review, 131, 435

Piskunov A. E., Schilbach E., Kharchenko N. V., Röser S., Scholz R.-D., 2008, A\&A, 477, 165

Plummer H. C., 1911, MNRAS, 71, 460

Podsiadlowski P., Langer N., Poelarends A. J. T., Rappaport S., Heger A., Pfahl E., 2004, ApJ, 612, 1044

Podsiadlowski P., Pfahl E., Rappaport S., 2005, in Rasio F. A., Stairs I. H., eds, Astronomical Society of the Pacific Conference Series Vol. 328, Binary Radio Pulsars. p. 327

Portegies Zwart S. F., McMillan S. L. W., Gieles M., 2010, ARA\&A, 48, 431

Postnov K., Kuranov A., 2017, preprint, (arXiv:1706.00369)

Postnov K. A., Yungelson L. R., 2014, Living Reviews in Relativity, 17,3

Repetto S., Davies M. B., Sigurdsson S., 2012, MNRAS, 425, 2799

Rodriguez C. L., Morscher M., Pattabiraman B., Chatterjee S., Haster C.-J., Rasio F. A., 2015, Physical Review Letters, 115, 051101

Rodriguez C. L., Chatterjee S., Rasio F. A., 2016a, Phys. Rev. D, 93, 084029

Rodriguez C. L., Haster C.-J., Chatterjee S., Kalogera V., Rasio F. A., 2016b, ApJ, 824, L8

Rodriguez C. L., Amaro-Seoane P., Chatterjee S., Rasio F. A., 2018, Physical Review Letters, 120, 151101

Spera M., Mapelli M., 2017, preprint, (arXiv:1706.06109)

Spera M., Mapelli M., Bressan A., 2015, MNRAS, 451, 4086

Spitzer L., 1987, Dynamical evolution of globular clusters

Stone N. C., Metzger B. D., 2016, MNRAS, 455, 859

Stone N. C., van Velzen S., 2016, ApJ, 825, L14

Stone N. C., Küpper A. H. W., Ostriker J. P., 2017, MNRAS, 467,4180

The LIGO Scientific Collaboration et al., 2017, preprint, (arXiv: 1711.05578)

Tutukov A. V., Cherepashchuk A. M., 2017, Astronomy Reports, 61,833

Tutukov A., Yungelson L., 1973, Nauchnye Informatsii, 27, 70

Zampieri L., Roberts T. P., 2009, MNRAS, 400, 677

Ziosi B. M., Mapelli M., Branchesi M., Tormen G., 2014, MNRAS, 441, 3703 